Geophysical and Astrophysical Fluid Dynamics

Vol. 00, No. 00, 00 Month 20xx, 1-21

\title{
Self-similar collapse of three geophysical vortices
}

\author{
Jean N. Reinaud \\ (Received 00 Month 20xx; final version received 00 Month 20xx)
}

\begin{abstract}
The self-similar collapse of three vortices is the motion of three vortices colliding at a single point at finite time. Such a motion has first been shown to exist for two-dimensional, planar, point vortices. In this paper we show that the concept generalises naturally to three-dimensional quasi-geostrophic vortices as well as to surface quasi-geostrophic vortices. We first determine the conditions which lead to the collapse for these singular vortices. We then show how these conditions precipitate the merger of finite core vortices both in a three-dimensional quasi-geostrophic flow and in a surface quasi-geostrophic flow.
\end{abstract}

\section{Introduction}

Vortices, or swirling masses of fluids are key dynamical features of the oceans and of the atmosphere. Zhang et al. (2014) have shown that they contribute to a significant part of the transport of mass in the oceans. Vortices interact with bathymetry, coasts, currents and also with other vortices. Like-signed vortices can merge to form larger structures if they are close enough to each other. Vortex merger is normally accompanied by the generation of small scale debris and filaments as a consequence of the conservation of energy and angular impulse. These feed both the inverse energy cascade and the direct enstrophy cascade in the geophysical turbulence discussed by Charney (1971) and Nastrom et al. (1984). The merger of two like-signed vortices may be precipitated by the influence of a third, opposite-signed vortex in their vicinity as discussed, for example, by Rodríguez-Marroyo et al. (2011).

The motion of three planar point vortices in a two-dimensional flow was first studied by Gröbli (1877). Synge (1949) further analysed the problem and provided a first classification of the motion. In particular, the author indicated the existence of contracting or expanding configurations of three vortices with a fixed shape. The problem was independently revisited by Novikov (1975) and by Aref (1979). Aref (1979) also showed the existence of configurations of three planar point vortices contracting to their centre of vorticity. This class of motion is hereinafter referred to as vortex collapse. Similar results were found by Tavantzis and Ting (1988). Additionally, Kimura (1987) explicitly studied the self-similar motion of three planar point vortices, providing a classification for such motions. In particular the author provided the conditions for collapse. Finally, Aref (2010) revisited the problem and included a stability analysis of the configurations.

The present paper is inspired by the studies by Kimura (1987) and Aref (2010) and generalises them to the motion of three point vortices in a three-dimensional quasi-geostrophic flow, focusing solely on the conditions leading to vortex collapse. The present study also provides the conditions for the collapse of point vortices in a pseudo two-dimensional, surface quasi-geostrophic flow.

Following the procedure described by Aref (2010), we find the necessary conditions for the self-similar motion of the point vortices, and we deduce the conditions for vortex collapse. We then describe in more details the collapse of point vortices when the two like-signed vortices have the same strength, before providing a rapid overview of the general case. We next investigate the interaction of three finite core vortices under initial conditions which 
lead to the collapse of the equivalent point vortices. To that end, we perform high resolution simulations for both for three-dimensional finite volume quasi-geostrophic vortices and for 'two-dimensional' surface quasi-geostrophic vortices. We show that the interaction may result in the gathering of the three vortices and the merger of the two like-signed vortices.

\section{Mathematical set-up}

Large scale oceanic and atmospheric flows are strongly influenced by the Earth's rotation and by the stable density stratification of the fluid. When $F r^{2} \ll R o \ll 1$, the flow evolution is accurately captured by the quasi-geostrophic (QG) model, which derives from an asymptotic expansion in Ro of Euler's equations. Here, $F r=U /(N H)$ and $R o=U /(f L)$ are the Froude and Rossby numbers respectively. $U$ is a horizontal velocity scale, $N$ is the buoyancy frequency, $f$ is the Coriolis frequency, and $H$ and $L$ are vertical and horizontal length scales respectively. We take $f$ and $N$ constant for simplicity. We stretch the vertical direction by the constant factor $f / N$. In this stretched reference frame, the QG potential vorticity anomaly $q$, hereinafter referred to as PV for simplicity, is defined from the flow streamfunction $\varphi$ as

$$
q=\frac{\partial^{2} \varphi}{\partial x^{2}}+\frac{\partial^{2} \varphi}{\partial y^{2}}+\frac{\partial^{2} \varphi}{\partial z^{2}} .
$$

The streamfunction $\varphi$ gives the horizontal advective (geostrophic) velocity components $u, v$ as well as the scaled buoyancy anomaly $\tilde{b}=b / N$,

$$
u=-\frac{\partial \varphi}{\partial y}, \quad v=\frac{\partial \varphi}{\partial x}, \quad \tilde{b}=\frac{\partial \varphi}{\partial z} .
$$

In the text, we refer to $\tilde{b}$ as buoyancy for simplicity. While the vertical velocity $w$ is not zero in the QG model, it is too small to contribute to the advection of PV. For an inviscid fluid in an adiabatic evolution, $\mathrm{PV}$ is materially conserved,

$$
\frac{\partial q}{\partial t}+u \frac{\partial q}{\partial x}+v \frac{\partial q}{\partial y}=0
$$

A full derivation of the QG model can be found in Vallis (2006). Equations (1), (2) and (3), with the addition of suitable boundary conditions, form a closed system of equations. In this work we consider two setups. In the first one, the flow is unbounded in all three dimensions of space and the boundary conditions are set by imposing $\varphi \rightarrow 0$ as $r=\sqrt{x^{2}+y^{2}+z^{2}} \rightarrow \infty$. Hereinafter, we refer to this setup as the three-dimensional QG case for simplicity. Importantly, a point vortex of strength $\kappa_{q}$ located at $\left(x_{0}, y_{0}, z_{0}\right)$ induces the streamfunction

$$
\varphi(x, y, z)=-\frac{\kappa_{q}}{\sqrt{\left(x-x_{0}\right)^{2}+\left(y-y_{0}\right)^{2}+\left(z-z_{0}\right)^{2}}},
$$

see for example Reinaud (2019). The strength $\kappa_{q}=(4 \pi)^{-1} \iiint q \mathrm{~d}^{3} \boldsymbol{x}$ of the point vortex has the dimension of a volume integrated PV.

The second setup also formally derives from the same general QG model, and is known as the surface quasi-geostrophic (SQG) model. In this model, the flow domain is semi-infinite and occupies, by convention, the upper half-domain $z \geq 0$. For oceanic applications, the same model can be derived for $z \leq 0$. Only the sign of the spectral inversion relation between the streamfunction and the buoyancy field differs between the two cases. In the SQG model, $q=0$ in the fluid interior and $\varphi$ is therefore harmonic. A distribution of buoyancy $\tilde{b}$ is prescribed at $z=0$ as Neumann boundary condition. Buoyancy is also materially conserved 


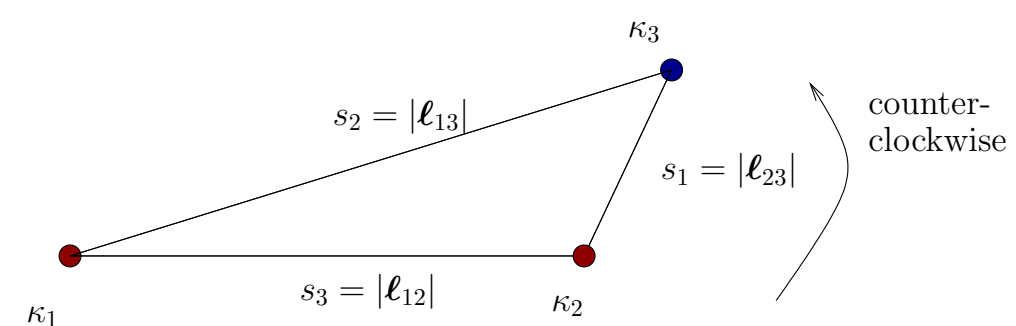

Figure 1. Geometry for the three vortices.

$$
\frac{\partial \tilde{b}}{\partial t}+u \frac{\partial \tilde{b}}{\partial x}+v \frac{\partial \tilde{b}}{\partial y}=0 \quad \text { at } \quad z=0
$$

Although the SQG model provides a flow in a three-dimensional fluid domain, one only needs to solve the equations at the surface $z=0$, hence the model can be mathematically seen as two-dimensional. Lapeyre (2017) provides a recent review of the SQG model. Importantly, an SQG 'point vortex' of strength $\kappa_{b}$ located $\left(x_{0}, y_{0}, 0\right)$ i.e. at the surface $z=0$, induces at the surface $z=0$ a streamfunction

$$
\varphi(x, y, 0)=-\frac{\kappa_{b}}{\sqrt{\left(x-x_{0}\right)^{2}+\left(y-y_{0}\right)^{2}}},
$$

see Held et al. (1995). The strength $\kappa_{b}=(2 \pi)^{-1} \iint \tilde{b} \mathrm{~d}^{2} \boldsymbol{x}$ of the point vortex has the dimension of a surface integrated buoyancy.

\section{Self-similar collapse of three point vortices}

\subsection{General conditions}

We consider three point vortices of strength $\kappa_{i}$ located at $\boldsymbol{x}_{i}, i=1,2,3$. Due to the absence of vertical advection in the QG model, all three point vortices can only collapse to a single point if they lie on the same horizontal plane. In this case the streamfunction induced by a point vortex onto another point vortex is formally the same in both the QG and the SQG models, see equation (4) with $z=z_{0}$ and equation (6). The calculation that follows is therefore valid for both three-dimensional QG point vortices and two-dimensional SQG point vortices.

Without loss of generality, we take $\kappa_{1}$ and $\kappa_{2}$ positive and $\kappa_{3}$ negative and the three vortices $1,2,3$ appear counter-clockwise. We define the vectors $\boldsymbol{\ell}_{i j}=\left(\ell_{i j}^{x}, \ell_{i j}^{y}, \ell_{i j}^{z}\right)=\boldsymbol{x}_{i}-\boldsymbol{x}_{j}=\left(x_{i}-\right.$ $\left.x_{j}, y_{i}-y_{j}, z_{i}-z_{j}\right)$ which indicate the sides of the triangle formed by the vortices. Since the point vortices are on the same plane $z=$ constant, $\ell_{i j}^{z}=0, \forall i, j$. By symmetry, $\ell_{i j}=-\ell_{j i}$. To simplify notations and following Aref (2010), we denote $s_{1}=\left|\ell_{23}\right|, s_{2}=\left|\ell_{13}\right|, s_{3}=\left|\ell_{12}\right|$, i.e. $s_{i}$ is the length of the side of the triangle opposing vortex $i$. The general geometry of the problem is described in figure 1.

The velocity of point vortex $i$ is given by

$$
\frac{\mathrm{d} \boldsymbol{x}_{i}}{\mathrm{~d} t}=\sum_{j, j \neq i} \frac{\kappa_{j}}{\left|\boldsymbol{x}_{i}-\boldsymbol{x}_{j}\right|^{3}}\left(-\left(y_{i}-y_{j}\right), x_{i}-x_{j}\right)=\sum_{j, j \neq i} \frac{\kappa_{j}}{\left|\ell_{i j}\right|^{3}}\left(-\ell_{i j}^{y}, \ell_{i j}^{x}\right) .
$$

We then deduce 


$$
\begin{aligned}
\frac{\mathrm{d} \ell_{23}}{\mathrm{~d} t} & =\frac{\mathrm{d} \boldsymbol{x}_{2}}{\mathrm{~d} t}-\frac{\mathrm{d} \boldsymbol{x}_{3}}{\mathrm{~d} t}, \\
& =\frac{\kappa_{1}}{s_{3}^{3}}\left(\ell_{12}^{y},-\ell_{12}^{x}\right)+\frac{\kappa_{3}}{s_{1}^{3}}\left(-\ell_{23}^{y}, \ell_{23}^{x}\right)+\frac{\kappa_{1}}{s_{2}^{3}}\left(-\ell_{13}^{y}, \ell_{13}^{x}\right)+\frac{\kappa_{2}}{s_{1}^{3}}\left(-\ell_{23}^{y}, \ell_{23}^{x}\right) .
\end{aligned}
$$

Taking the scalar product of equation (8) with $\boldsymbol{\ell}_{23}$, we obtain, after simplification,

$$
\ell_{23} \cdot \frac{\mathrm{d} \ell_{23}}{\mathrm{~d} t}=-\frac{\kappa_{1}}{s_{3}^{3}}\left(\ell_{12}^{x} \ell_{23}^{y}-\ell_{12}^{y} \ell_{23}^{x}\right)+\frac{\kappa_{1}}{s_{2}^{3}}\left(\ell_{13}^{x} \ell_{23}^{y}-\ell_{13}^{y} \ell_{23}^{x}\right)
$$

Next we use the fact that

$$
\begin{aligned}
\Delta & =\ell_{12}^{x} \ell_{23}^{y}-\ell_{12}^{y} \ell_{23}^{x}=\ell_{12} \times \ell_{23} \cdot \boldsymbol{k}=\left|\ell_{12} \times \ell_{23}\right|, \\
& =\ell_{13}^{x} \ell_{23}^{y}-\ell_{13}^{y} \ell_{23}^{x}=\ell_{13} \times \ell_{23} \cdot \boldsymbol{k}=\left|\ell_{13} \times \ell_{23}\right|,
\end{aligned}
$$

is twice the area of the counter-clockwise vortex triangle 123 to obtain

$$
\frac{\mathrm{d} s_{1}^{2}}{\mathrm{~d} t}=2 \kappa_{1} \Delta\left(\frac{1}{s_{2}^{3}}-\frac{1}{s_{3}^{3}}\right) .
$$

It should be noted that the factor $\Delta$ defined here differs by a factor two from the one used by Aref (2010).

Similarly we have,

$$
\begin{aligned}
& \frac{\mathrm{d} s_{2}^{2}}{\mathrm{~d} t}=2 \kappa_{2} \Delta\left(\frac{1}{s_{3}^{3}}-\frac{1}{s_{1}^{3}}\right), \\
& \frac{\mathrm{d} s_{3}^{2}}{\mathrm{~d} t}=2 \kappa_{3} \Delta\left(\frac{1}{s_{1}^{3}}-\frac{1}{s_{2}^{3}}\right),
\end{aligned}
$$

for the two other sides.

Equations (12), (13) and (14) are formally similar to the ones obtained for two-dimensional, planar point vortices. The main difference is that the terms in brackets are in $s_{i}^{-3}$ for QG/SQG vortices compared to $s_{i}^{-2}$ for two-dimensional, planar point vortices.

We next look for a self-similar solution in the form

$$
s_{i}(t)=f(t) s_{i}(0) .
$$

Again, due to the lack of vertical advection, the self-similar motion is only possible if the vortices lie on the same horizontal plane. Otherwise $s_{i}(t)=$ $\sqrt{\left.\left(x_{j}(t)-x_{k}(t)\right)^{2}+\left(y_{j}(t)-y_{k}(t)\right)^{2}\right)+\left(z_{j}-z_{k}\right)^{2}}$ would include a constant term $z_{j}-z_{k}$. Using Heron's formula for the area of a triangle, $4 \Delta^{2}=2\left(s_{1}^{2} s_{2}^{2}+s_{1}^{2} s_{3}^{2}+s_{2}^{2} s_{3}^{2}\right)-s_{1}^{4}-s_{2}^{2}-s_{3}^{4}$, we see that $\Delta(t)$ is proportional to $f^{2}(t)$. Substituting equation (15) into equation (12), we obtain

$$
\frac{\mathrm{d} f^{2}}{\mathrm{~d} t}=2 \kappa_{1} \frac{\Delta(0)}{s_{1}^{2}(0)}\left(\frac{1}{s_{2}^{3}(0)}-\frac{1}{s_{3}^{3}(0)}\right) \frac{1}{f(t)},
$$

which gives

$$
f(t)=\sqrt[3]{1-\frac{t}{\tau}}
$$

where 


$$
\tau=-\frac{s_{1}^{2}(0) s_{2}^{3}(0) s_{3}^{3}(0)}{3 \kappa_{1} \Delta(0)\left(s_{3}^{3}(0)-s_{2}^{3}(0)\right)},
$$

using $f(0)=1$. The form of $f$ justifies the self-similar collapse of the three vortices at finite time $\tau$ for $\tau>0$. It should be noted that case $f(t)=1=$ constant for $s_{1}(0)=s_{2}(0)=s_{3}(0)$ is also solution. It is recovered by our general solution and corresponds to $\tau \rightarrow \infty$. This case therefore naturally appears as a limiting case in our study and is further discussed in the paper.

Again, the solution is similar to the one obtained for two-dimensional, planar point vortices, except that the time-dependence is a cubic root for the QG/SQG vortices compare to a square root for the classic planar vortices.

Since equations (12), (13) and (14) must lead to the same value of $\tau$, the existence of the self-similar solution implies

$$
\kappa_{1} s_{1}(0)\left(s_{3}^{3}(0)-s_{2}^{3}(0)\right)=\kappa_{2} s_{2}(0)\left(s_{1}^{3}(0)-s_{3}^{3}(0)\right)=\kappa_{3} s_{3}(0)\left(s_{2}^{3}(0)-s_{1}^{3}(0)\right) .
$$

As for the planar point vortex case, the linear impulse

$$
\boldsymbol{I}=\left(I_{x}, I_{y}, I_{z}\right)=\kappa_{1} \boldsymbol{x}_{1}+\kappa_{2} \boldsymbol{x}_{2}+\kappa_{3} \boldsymbol{x}_{3},
$$

and the angular impulse

$$
J=\kappa_{1}\left(x_{1}^{2}+y_{1}^{2}\right)+\kappa_{2}\left(x_{2}^{2}+y_{2}^{2}\right)+\kappa_{3}\left(x_{3}^{2}+y_{3}^{2}\right)
$$

are conserved quantities.

Additionally the Hamiltonian of a system of $N$ point vortices may be defined as

$$
H=\frac{1}{2} \sum_{i=1}^{N} \sum_{\substack{j=1 \\ j \neq i}}^{N} \frac{\kappa_{i} \kappa_{j}}{\left|\ell_{i j}\right|}
$$

and is also an invariant of the motion, see for example Dritschel et al. (2004). Here, we have

$$
H=\frac{\kappa_{1} \kappa_{2}}{s_{3}}+\frac{\kappa_{1} \kappa_{3}}{s_{2}}+\frac{\kappa_{2} \kappa_{3}}{s_{1}}
$$

The invariance of $H$ means $H(t)=H(0)$, where $H(t)$ is the value of the Hamiltonian evaluated at time $t$ and $H(0)$ is the value of the Hamiltonian evaluated at time $t=0$. This implies

$$
\frac{\kappa_{1} \kappa_{2}}{s_{3}(t)}+\frac{\kappa_{1} \kappa_{3}}{s_{2}(t)}+\frac{\kappa_{2} \kappa_{3}}{s_{1}(t)}=\frac{1}{f(t)}\left(\frac{\kappa_{1} \kappa_{2}}{s_{3}(0)}+\frac{\kappa_{1} \kappa_{3}}{s_{2}(0)}+\frac{\kappa_{2} \kappa_{3}}{s_{1}(0)}\right)=\frac{\kappa_{1} \kappa_{2}}{s_{3}(0)}+\frac{\kappa_{1} \kappa_{3}}{s_{2}(0)}+\frac{\kappa_{2} \kappa_{3}}{s_{1}(0)} .
$$

This is only possible if

$$
\frac{\kappa_{1} \kappa_{2}}{s_{3}(0)}+\frac{\kappa_{1} \kappa_{3}}{s_{2}(0)}+\frac{\kappa_{2} \kappa_{3}}{s_{1}(0)}=0 .
$$

It should be noted that the condition (25), imposed by the invariance of $H$, not only depends on the vortex strengths $\kappa_{i}$ but also on the distances $s_{i}(0)$ separating them at $t=0$. This is in contrast with the classic planar case where the constraint is on the vortex strengths alone, see Aref (2010).

Following Aref (2010), we introduce the invariant $L$, a combination of the invariants $J$ and $\boldsymbol{I}$ defined as

$$
L=\left(\kappa_{1}+\kappa_{2}+\kappa_{3}\right) J-I_{x}^{2}-I_{y}^{2} .
$$


As for the invariance of $H$, the invariance of $L, L(t)=L(0)$, implies that

$$
\begin{aligned}
L & =\kappa_{1} \kappa_{2} s_{3}^{2}(t)+\kappa_{1} \kappa_{3} s_{2}^{2}(t)+\kappa_{2} \kappa_{3} s_{1}^{2}(t), \\
& =f^{2}(t)\left(\kappa_{1} \kappa_{2} s_{3}^{2}(0)+\kappa_{1} \kappa_{3} s_{2}^{2}(0)+\kappa_{2} \kappa_{3} s_{1}^{2}(0)\right), \\
& =\kappa_{1} \kappa_{2} s_{3}^{2}(0)+\kappa_{1} \kappa_{3} s_{2}^{2}(0)+\kappa_{2} \kappa_{3} s_{1}^{2}(0) .
\end{aligned}
$$

This is only possible if

$$
\kappa_{1} \kappa_{2} s_{3}^{2}(0)+\kappa_{1} \kappa_{3} s_{2}^{2}(0)+\kappa_{2} \kappa_{3} s_{1}^{2}(0)=0 .
$$

This condition imposed by the invariance of $L$ is formally the same as in the classic planar case, see Aref (2010).

It is straightforward to show that equations (25) and (28) ensure that equation (19) is also satisfied. Indeed, expressing $\kappa_{1} \kappa_{2} s_{3}^{2}$ using equation (25) gives

$$
\kappa_{1} \kappa_{2} s_{3}^{2}(0)=-s_{3}^{3}(0)\left(\frac{\kappa_{1} \kappa_{3}}{s_{2}(0)}+\frac{\kappa_{2} \kappa_{3}}{s_{1}(0)}\right),
$$

while equation (28) gives

$$
\kappa_{1} \kappa_{2} s_{3}^{2}(0)=-\kappa_{1} \kappa_{3} s_{2}^{2}(0)-\kappa_{2} \kappa_{3} s_{1}^{2}(0),
$$

Equating the two and re-arranging, we obtain

$$
\kappa_{1}\left(s_{2}^{2}(0)-\frac{s_{3}^{3}(0)}{s_{2}(0)}\right)=\kappa_{2}\left(\frac{s_{3}^{3}(0)}{s_{1}(0)}-s_{1}^{2}(0)\right),
$$

which proves the first equality in equation (19). The second equality can be proved in a similar way. We therefore have two necessary conditions for the self-similar motion, given by equations (25) and (28). The final condition for the vortex collapse is to impose that $\tau$ is finite and positive.

\section{2. $\quad$ Case $\kappa_{1}=\kappa_{2}$}

We next detail the conditions for the self-similar collapse of three vortices when the two likesigned vortices have equal strength. This is an important special case since the merger of two finite-core like-signed vortices often occurs between two vortices of comparable strengths. The strong interaction between two finite-core vortices of largely unequal strength may lead to the partial or complete straining out of the vortex of smaller strength. Although such interactions are of interest, we mainly focus in the rest of the paper on the conditions precipitating vortex merger.

We set $\kappa_{1}=\kappa_{2}=1$ and $s_{3}(0)=1$ without loss of generality. The collapse occurs when $\tau>0$. This implies $\kappa_{3}<0$ and $s_{1}(0)<s_{3}(0)<s_{2}(0)$. Recall that the point vortices appear counterclockwise. This implies that $\Delta(0)$ is positive. We still have three parameters to determine, namely $s_{1}(0), s_{2}(0)$ and $\kappa_{3}$ and two conditions to impose. We therefore parametrise the solution by $s_{1}(0)=s \in(0,1)$. Equations $(25)$ and $(28)$ become

$$
\begin{aligned}
1+\kappa_{3}\left(\frac{1}{s}+\frac{1}{s_{2}(0)}\right) & =0, \\
1+\kappa_{3}\left(s^{2}+s_{2}^{2}(0)\right) & =0
\end{aligned}
$$

Eliminating $\kappa_{3}$, we obtain a cubic equation for $s_{2}(0)$,

$$
s_{2}^{3}(0)+\frac{s^{3}-1}{s} s_{2}(0)-1=0
$$




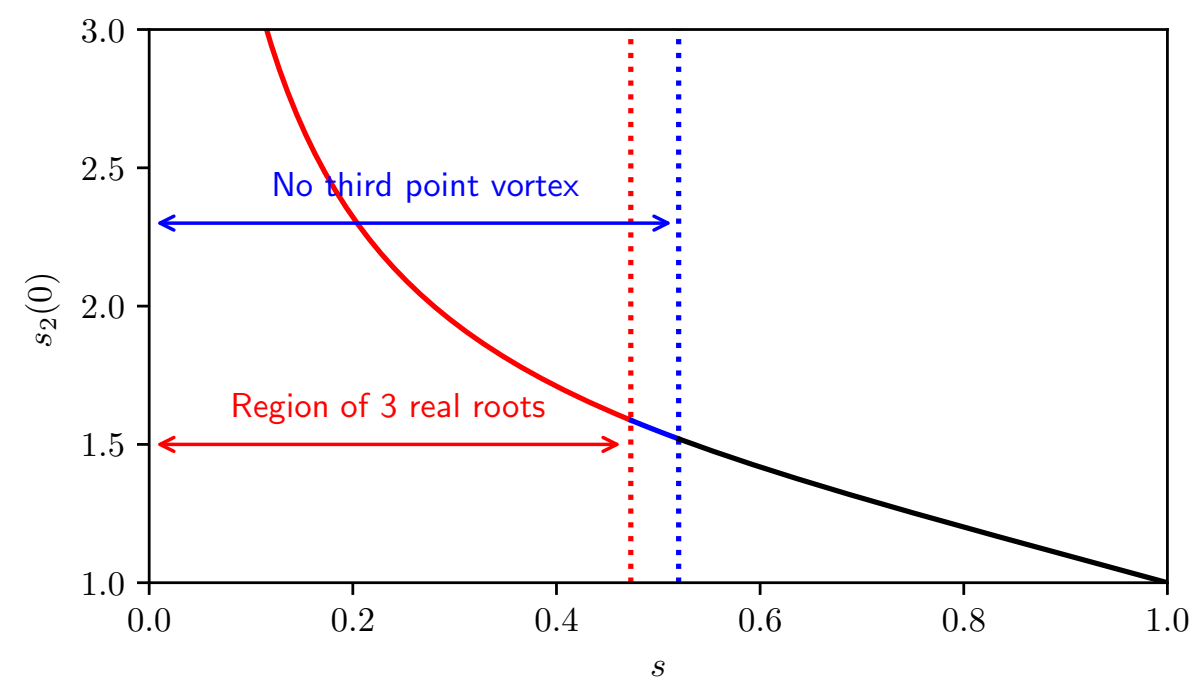

Figure 2. Root $s_{2}(0)>1$ given by equation (34). The region in red corresponds to the region where the equation (33) has 3 real roots. Only one of these roots is positive and physically relevant. The region is blue corresponds to the region where the distances $s=s_{1}(0)$ and $s_{2}(0)$ do not lead to no real solution for the location of vortex 3 (colour online).

This cubic equation (33) has either three real roots or a single real root. First, recall that the root $s_{2}(0)>0$ as $s_{2}$ is a length. In our case, only the region where the equation has a single real root leads to a physically acceptable solution as we will justify later. In this case the real root is

$$
s_{2}(0)=\sqrt[3]{\frac{1}{2}+\sqrt{\frac{1}{4}+\frac{1}{27}\left(\frac{s^{3}-1}{s}\right)^{3}}}+\sqrt[3]{\frac{1}{2}-\sqrt{\frac{1}{4}+\frac{1}{27}\left(\frac{s^{3}-1}{s}\right)^{3}}}
$$

provided

$$
\left(\frac{s^{3}-1}{s}\right)^{3} \geq-\frac{27}{4}
$$

or $s>S_{m} \simeq 0.4731$. Note that the formula is still valid and provides one of the three real solutions if $s<S_{m}$ and if the calculation is performed in $\mathbb{C}$.

Figure 2 gives the root $s_{2}(0)>1$ of equation $(34)$ for $s \in(0,1)$. We note that the limiting case $s \rightarrow 1^{-}$corresponds to $s_{2}(0) \rightarrow 1^{+}$, hence the vortex triangle is isosceles, and the configuration tends to a steady state (an infinitely slow collapse with $\tau \rightarrow \infty$ ). The strength of the third vortex $\kappa_{3}$ can be found by using either equation (25) or equation (28). It is important to recall that $\kappa_{3}$ depends not only on $\kappa_{1}$ and $\kappa_{2}$, but formally also on $s_{3}(0)$ and $s=s_{1}(0)$. This is in contrast with the collapse of planar point vortices where $\kappa_{3}$ is completely determined by $\kappa_{1}$ and $\kappa_{2}$. The value of $s_{2}(0)$ found by solving equation (33) however only provides a physically acceptable solution if it corresponds to the real location for the point vortex 3. Without loss of generality, we place vortex 1 at $(-0.5,0,0)$ and vortex 2 at $(0.5,0,0)$ at $t=0$. At $t=0$, vortex 3 is at a distance $s=s_{1}(0)=\left|\ell_{23}\right|$ from vortex 2 and $s_{2}(0)=\left|\ell_{13}\right|$ from vortex 1 , and is located at $\left(x_{3}(0), y_{3}(0), 0\right)$ such that

$$
\begin{gathered}
\left(x_{3}(0)-0.5\right)^{2}+y_{3}^{2}(0)=s^{2}, \\
\left(x_{3}(0)+0.5\right)^{2}+y_{3}^{2}(0)=s_{2}^{2}(0) .
\end{gathered}
$$


Subtracting the two equations we obtain

$$
x_{3}(0)=\frac{s_{2}^{2}(0)-s^{2}}{2}>0,
$$

and

$$
y_{3}(0)=\sqrt{s^{2}-\left(x_{3}(0)-0.5\right)^{2}}>0,
$$

for a counter-clockwise vortex triangle 123 . The solution is physical only if $y_{3}(0) \in \mathbb{R}$, i.e. $\left|x_{3}(0)-0.5\right|<s$. This imposes a second lower bound $S_{c}$ on $s$. Indeed the circle of radius $s$ centred at vortex 2 , and the circle of radius $s_{2}(0)$ centred around vortex 1 must intersect for the position of vortex 3 to be defined. If they do not, there is no possible location for vortex 3 for self-similar collapse, hence self-similar collapse is not possible. The lower bound $S_{c}$ can formally be obtained by solving the nontrivial implicit equation for $s$ obtained by imposing $y_{3}^{2}(0)=0$ from equation (38) where $x_{3}(0)$ is given by equation (37) and $s_{2}(0)$ is given by equation (34). For $\kappa_{1}=\kappa_{2}=1$, and $s_{3}(0)=1$ this imposes $s>S_{c} \simeq 0.520021>S_{m}$.

Figure 3 describes the strength $\kappa_{3}$ and the initial location $\left(x_{3}(0), y_{3}(0), 0\right)$ of the third, opposite-signed vortex, parametrised by the distance $s=s_{1}(0)$ for $\kappa_{1}=\kappa_{2}=1$, and $s_{3}(0)=1$. It is interesting to notice that $\left|\kappa_{3}\right|<\kappa_{1}$ in all cases. The opposite-signed vortex is typically weaker than the two equal-strength vortices. Similar results can be obtained for other ratios $\kappa_{2} / \kappa_{1}$ and are briefly addressed in subsection 3.3.

Figure 4 provides the time $\tau$ for the vortex collapse for $\kappa_{1}=\kappa_{2}=1$ and $s_{3}(0)=1$ as a function of $s$. $\tau$ reaches a minimum $\tau \simeq 0.3657$ for $s \simeq 0.609$. $\tau$ diverges rapidly to infinity as $s \rightarrow 1$. Indeed, as mentioned above, when $s \rightarrow 1$ the vortex triangle approaches the equilibrium, isosceles configuration and $\tau \propto\left(s_{3}^{2}(0)-s_{2}^{3}\right)^{-1} \rightarrow \infty . \tau \rightarrow \infty$ as $s \rightarrow S_{c} \simeq 0.520021$ as well. As mentioned above, this corresponds to the boundary of the domain where real solutions exists, i.e. to $y_{3} \rightarrow 0$ in equation (38). In this limit, all three vortices align along the $y$-axis, flattening the vortex triangle. Hence the area of the triangle $\Delta(0) \rightarrow 0$ resulting in $\tau \propto \Delta(0)^{-1} \rightarrow \infty$. Both cases $s \rightarrow 1$ and $s \rightarrow S_{c} \simeq 0.520021$ therefore correspond to equilibria, $\mathrm{d} s_{i} / \mathrm{d} t \rightarrow 0$ or $f(t) \rightarrow 1, \forall t$.

We next simulate the motion of the three point vortices for $s=0.6$ by integrating numerically explicitly equations $(7)$. In this case, $s_{2}(0) \simeq 1.4184$ and $\left(x_{3}(0), y_{3}(0), 0\right) \simeq$ $(0.8259,0.5038,0)$ with $\kappa_{3} \simeq-0.4216$. Note that the simulation is initialised by the values of $\kappa_{3}, x_{3}(0), y_{3}(0)$ obtained at machine precision. Equation (18) gives $\tau \simeq 0.36671$.

Time is marched using a fourth-order Runge-Kutta scheme with a time step controlled by the maximum velocity of the point vortices. Results are shown in figure 5 . The trajectory of the three point vortices, shown in figure $5(a)$, confirms the contracting, inward spiralling, motion of the point vortices. The evolution of the distances $s_{i}(t)$ separating the point vortices, shown in figure $5(b)$, suggests a self-similar behaviour, with the distances $s_{i}(t)$ collapsing to 0 at $t \simeq 0.3667$. This is further confirmed in figure $5(c)$ where it is shown that the length ratios $s_{1}(t) / s_{3}(t)$ and $s_{2}(t) / s_{3}(t)$ are constant in time. Finally by plotting $1-\left(s_{1}(t) / s_{1}(0)\right)^{3}$, we show that we recover the expected linear evolution $t / \tau$, confirming that $s_{i}(t) \propto \sqrt[3]{1-t / \tau}$.

\subsection{General case}

We next briefly discuss the general case. Without loss of generality we can keep $\kappa_{1}=1$, and $s_{3}(0)=1$. We now vary both $s_{1}(0) \in(0,1]$ and $\kappa_{2} \in(0,1]$. Results are presented in figure 6 . As for the special case $\kappa_{2}=\kappa_{1}$ discussed in section 3.2, real solutions for the location of the third vortex can only be found in a part of the parameter space. For each value of $\kappa_{2}$, there is a threshold $S_{c}$ for $s$ below which no solution exists. This threshold varies little as $\kappa_{2}$ varies. It decreases monotonically from $S_{c} \simeq 0.559$ for $\kappa_{2} \rightarrow 0$ to $S_{c} \simeq 0.5200$ for $\kappa_{2} \rightarrow 1$, a relative variation of $7 \%$ only. 

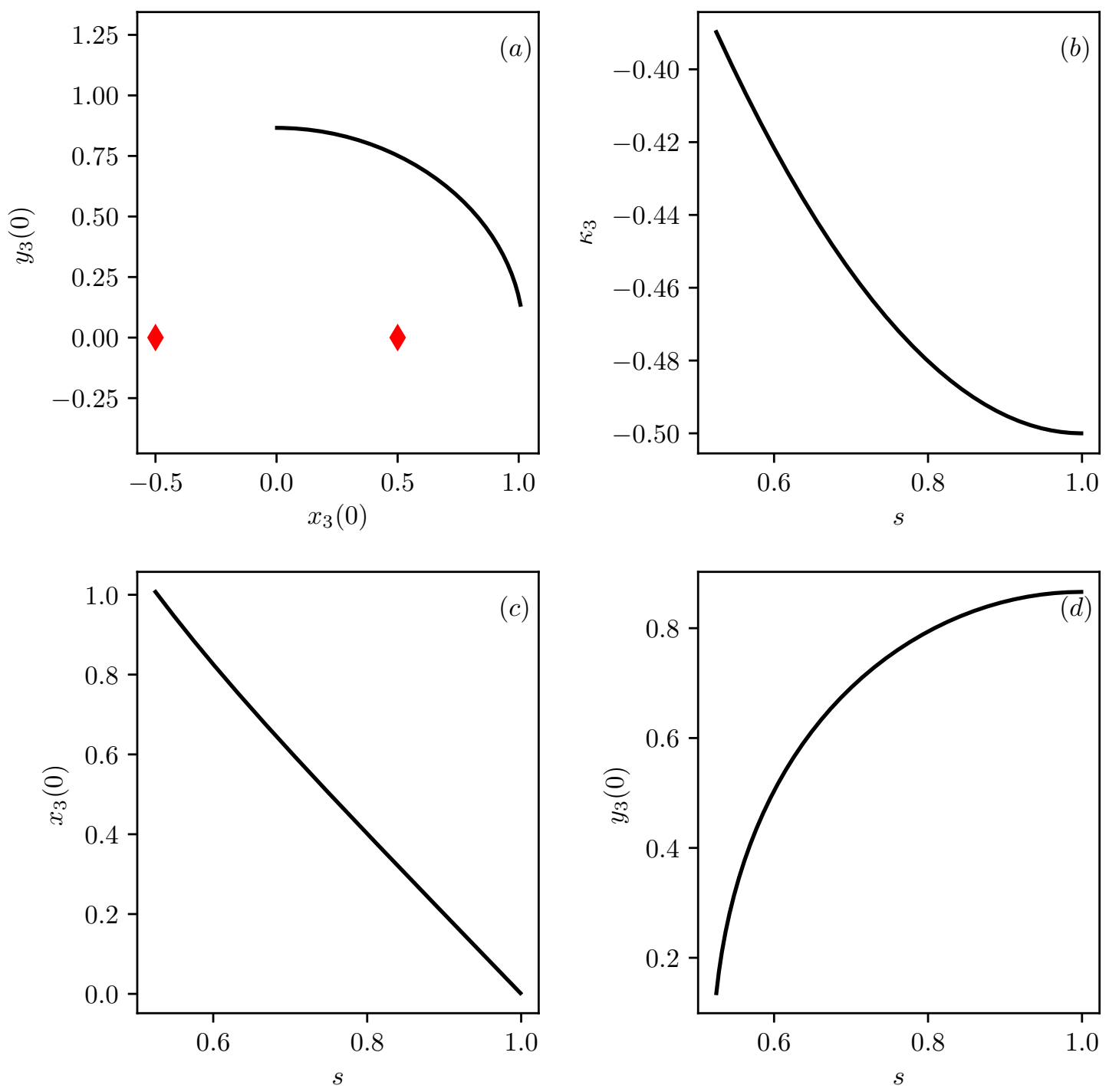

Figure 3. Conditions for the self-similar collapse of three point vortices for $\kappa_{1}=\kappa_{2}=1$ and $s_{3}(0)=1$, and for $s \in[0.521,0.999]$. (a): loci of the third vortex (black solid line). The location of vortex 1 and 2 is indicated by red diamonds. (b) Strength $\kappa_{3}$ vs $s=s_{1}(0)$. (c) $x$-coordinate $x_{3}(0)$ of the third vortex vs $s .(d) y$-coordinate $y_{3}(0)$ of the third vortex vs $s$ (colour online).

Overall, we also see that the distance $s_{2}(0)$ depends only weakly on $\kappa_{2}$. On the other hand $\kappa_{3}$ is nearly proportional to $\kappa_{2}$. Qualitatively, results are similar for all values of $\kappa_{2}$.

Contours of the time $\tau$ for the finite-time self-similar collapse of the three point vortices are presented in figure 7 in the $\left(s, \kappa_{2}\right)$-plane. Again, $\tau \rightarrow \infty$ as $s \rightarrow S_{c}$. The minimum of $\tau$, corresponding to the fastest self-similar collapse, is found for intermediate values of $s\left(\kappa_{2}\right)$, varying from $s \simeq 0.68$ as $\kappa_{2} \ll 1$ to $s \simeq 0.61$ as $\kappa_{2} \rightarrow 1$.

\section{Examples of the collapse for finite volume QG vortices}

We next use the strength and location of the three point vortices with $\kappa_{1}=\kappa_{2}$ and $s_{1}(0) / s_{3}(0)=0.6$, calculated for the self-similar collapse of the point vortices, to initialise numerical simulations for finite volume vortices. By considering finite volume vortices, we 

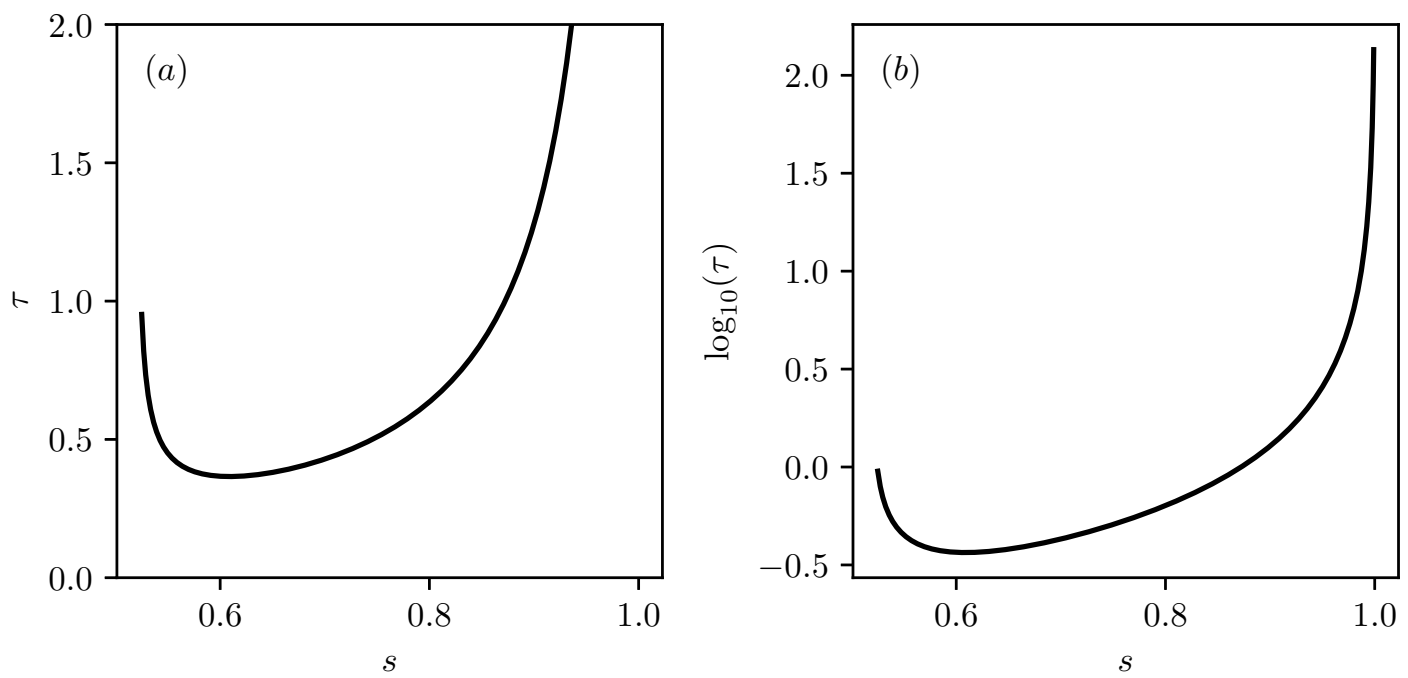

Figure 4. Time $\tau$ for the self-similar collapse of the three vortices with $\kappa_{1}=\kappa_{2}=1$ and $s_{3}(0)=1$ vs $s=s_{1}(0)$ for $s \in[0.521,0.999] .(a): \tau$ in the range $(0,2],(b): \log _{10}(\tau)$ to better see the rapid divergence of $\tau$ as $s \rightarrow S_{c}$ and $s \rightarrow 1$.

introduce a new length scale to the problem, associated with the vortex sizes. For the sake of simplicity, all three vortices have the same size in our numerical experiments. We perform two simulations. In the first one, the three vortices are initially spheres of uniform PV of identical radius $r_{0} / s_{3}(0)=0.2$. In the second one, we set $r_{0} / s_{3}(0)=0.1$. In both cases the equal PV of vortices 1 and 2 is set to $q_{1}=q_{2}=q_{0}=2 \pi$. The simulations are performed using the purely Lagrangian quasi-geostrophic Contour Dynamics algorithm in an explicitly unbounded domain. A description of the method is provided by Dritschel and Saravanan (1994). Vortices are mapped in the vertical direction by 200 layers. The complexity of the vortex bounding contours is controlled by Contour Surgery, introduced by Dritschel (1988). The two surgery parameters are the large-scale length $L=2 r_{0}$, and the dimensionless parameter $\mu=0.1$, such that the maximum node spacing along the contours is $\mu L$. The length scale of the problem is set by imposing that the total height of $\mathrm{PV}, H=2 r_{0}=1$, while the time scale is implicitly set by $q_{0}$. For reference, a single sphere of uniform PV $q_{0}$ has a turnover period of $T_{\text {over }}=6 \pi / q_{0}=3$ here. The strength of the two like-signed vortices is $\kappa_{1}=\kappa_{2}=q_{0} r_{0}^{3} / 3=\pi / 12$.

Snapshots of the flow for $r_{0} / s_{3}(0)=0.2$ are shown in figure 8 . As expected, the vortices get closer together. Contrarily to point vortices, finite volume vortices have a shape and therefore can deform when subjected to the strain induced by the other vortices. Hence a part of the energy of the flow is used for this deformation, slowing down the vortices. Since the velocity induced by a given vortex decays with the distance $d$ from the vortex as $1 / d^{2}$, the strain rate induced by a vortex decays as $1 / d^{3}$. As the vortices get closer together, the strain the vortices induce onto each other increases rapidly. At $t=20$, the two like-signed, cyclonic $\left(q_{1,2}>0\right)$, vortices merge. The opposite-signed, anticyclonic $\left(q_{3}<0\right)$ vortex is strongly deformed by the strain induced by the merged cyclone. At later times, the anticyclone is almost completely sheared out, and a large filament of positive PV is shed by the merged cyclones.

We next focus the early evolution of the flow, until the two like-signed vortices merge. We first determine the geometric centre $\boldsymbol{x}_{i}$ of each vortex at each time $t$ by contour integration. The trajectory of the three vortex centres is shown in figure $9(a)$ until $t=20$, when the two like-signed vortices merge. It confirms the initial inward spiralling of the vortices. As in the point vortex case, the evolution of the distances $s_{i}(t)$ separating the vortices, shown in figure $9(b)$ again suggests a self-similar contracting motion. We next define the self-similarity 

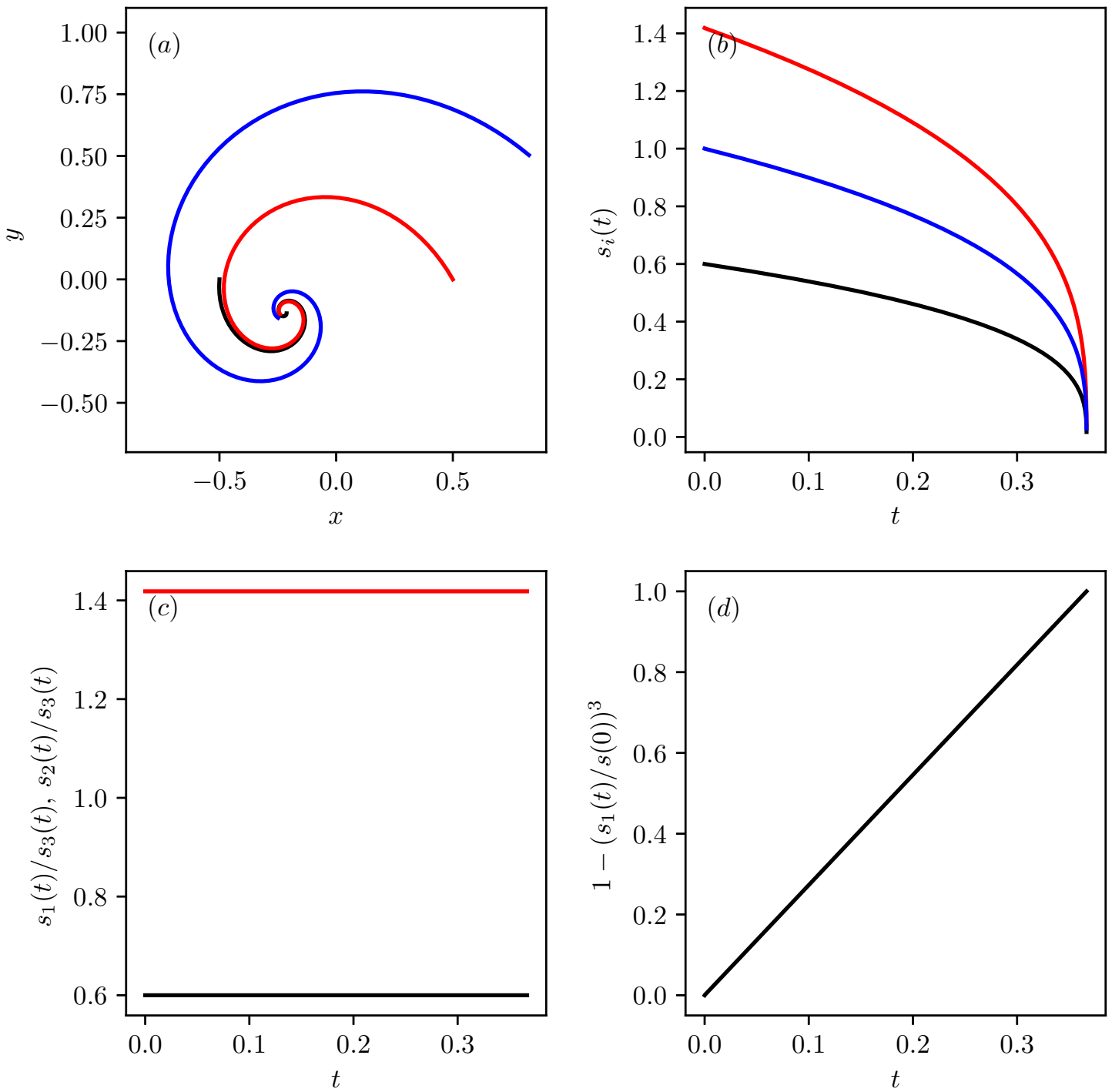

Figure 5. Evolution of three point vortices in self-similar collapse conditions. $(a)$ : Trajectory of the point vortices, $(b)$ evolution of the distances $s_{i}(t), i=1,2,3(c)$ evolution of the distance ratios $s_{1}(t) / s_{3}(t)$ (black) and $s_{2}(t) / s_{3}(t)($ red), $(d)$ similarity function $1-s_{1}(t) / s_{1}(0)=t / \tau$ for $\kappa_{1}=\kappa_{2}=1, s_{3}(0)=1$ and $s=s_{1}(0)=0.6$ to $t \in[0,0.3667]$ (colour online).

functions $g_{i}$ for vortex $i$

$$
g_{i}(t)=1-\left(\frac{s_{i}(t)}{s_{i}(0)}\right)^{3}
$$

in a similar way it was done for the point vortices. Recall that if the evolution is exactly selfsimilar, then the three functions $g_{i}(t)$ are in fact equal, with $g_{i}(t)=t / \tau, i=1,2,3$, i.e. linear functions with the same slope $1 / \tau$. Moreover, the functions $g_{i}(t)$ also allow to verify whether the self-similar motion follows a power law $\propto \sqrt[3]{1-t / \tau}$. The evolution of the self-similarity functions $g_{i}$ is shown in figure $9(c)$. Results indicate that, initially, the motion of the three vortex centres is self-similar as all three functions $g_{i}(t), i=1,2,3$ collapse to the same line. As the flow evolves however and the vortices deform, the evolution slowly departs from the exact self-similar evolution. This is first due to a cumulative effect of small differences between the 'rigid' point vortices and deformable finite core vortices, but also to the fact that deformation 

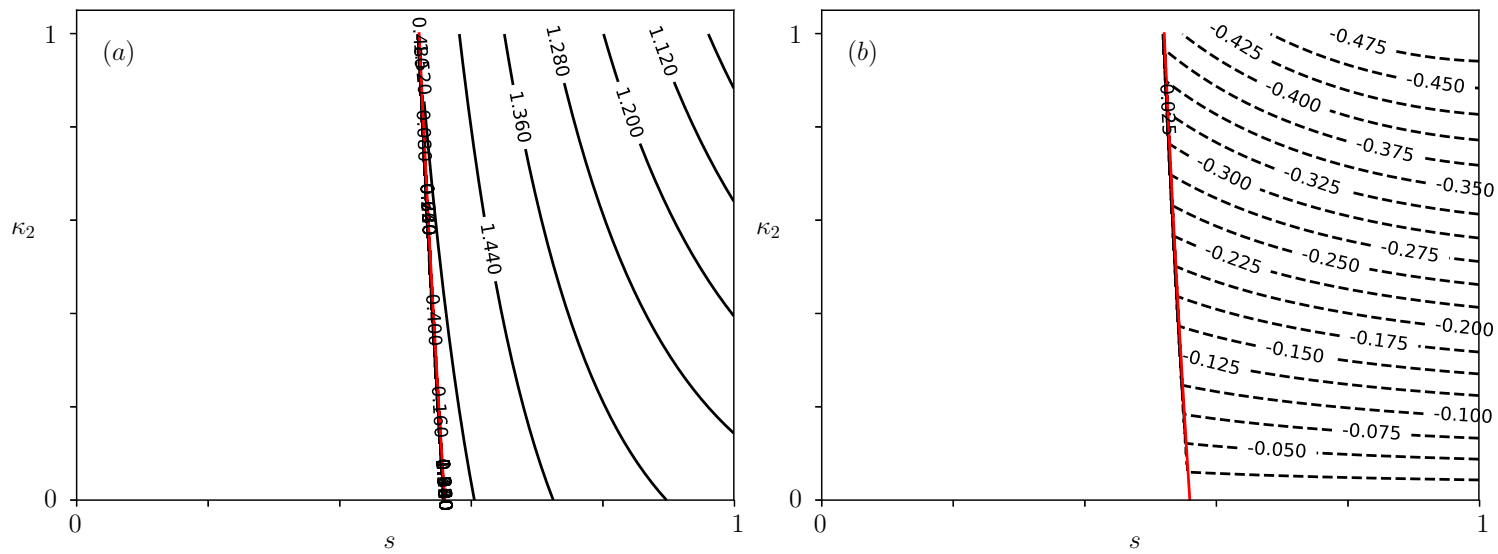

Figure 6. (a) Contours of the distance $s_{2}(0)$ and $(b)$ contours of the strength $\kappa_{3}$ of the third vortex for the self-similar vortex collapse for $\kappa_{1}=1$, and $s_{3}(0)=1$ in the parameter space $\left(s=s_{1}(0), \kappa_{2}\right)$. The solid red line indicates $S_{c}$ which delimits the regions where solutions exist (no solution on the left of the line; colour online).

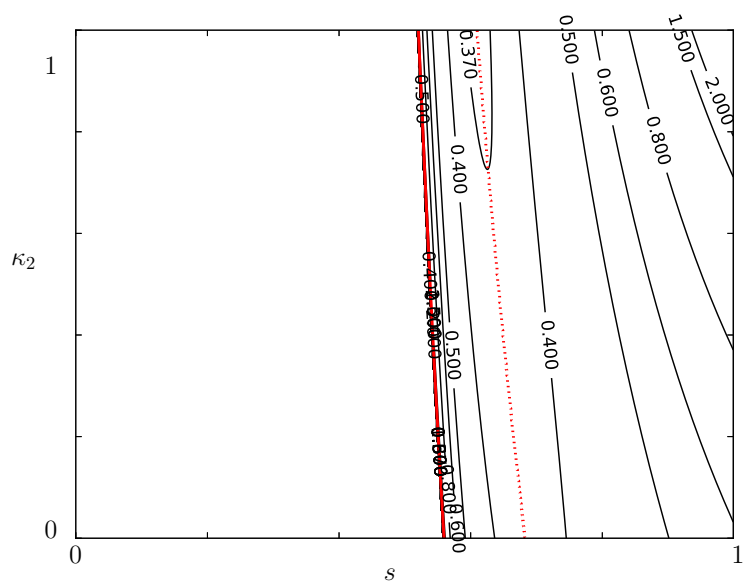

Figure 7. Contours of the time $\tau$ for the self-similar vortex collapse for $\kappa_{1}=1$, and $s_{3}=1$ in the parameter space $\left(s=s_{1}, \kappa_{2}\right)$. The red line delimits the regions where solutions exist (no solution on the left of the line). The dotted red line indicates the loci of the minimum for $\tau$ (colour online).

increases as the vortices get closer together. Yet, the curves $g_{i}(t)$ remains fairly linear and close to each other indicating a near self-similar evolution. The linearity of the curves also confirms that cubic-root power law for the motion. The upshot is that the presence of the opposite-signed vortex has precipitated the merger of the two like-signed vortices by making all three vortices move closer together.

In the next experiment $r_{0} / s_{3}(0)=0.1$. Snapshots of the flow are presented in figure 10 . The merger process is delayed compared to the previous case. Indeed, two like-signed vortices may merge provided the distance separating them is less than a threshold, proportional to the vortex radius, see for example Reinaud and Dritschel (2002) for the merger of two threedimensional QG vortices. By reducing the vortex radii, the vortices travel longer along the inward spiralling path before getting close enough together, compared to the previous case. The two like-signed vortices merge by $t=183.5$ to form a dumbbell like structure. This structure eventually breaks asymmetrically. This is a case of partial merger, often seen for the binary interaction between two like-signed quasi-geostrophic vortices, see Reinaud and Dritschel (2002).

Figure 11 shows the trajectories of the vortex centres, the evolution of the distances $s_{i}(t)$ and the self-similarity functions $g_{i}(t)$ for this case. Results show that when starting with vortices with a smaller relative radius $r_{0} / s_{3}(0)$, the vortices have an early evolution closer to 

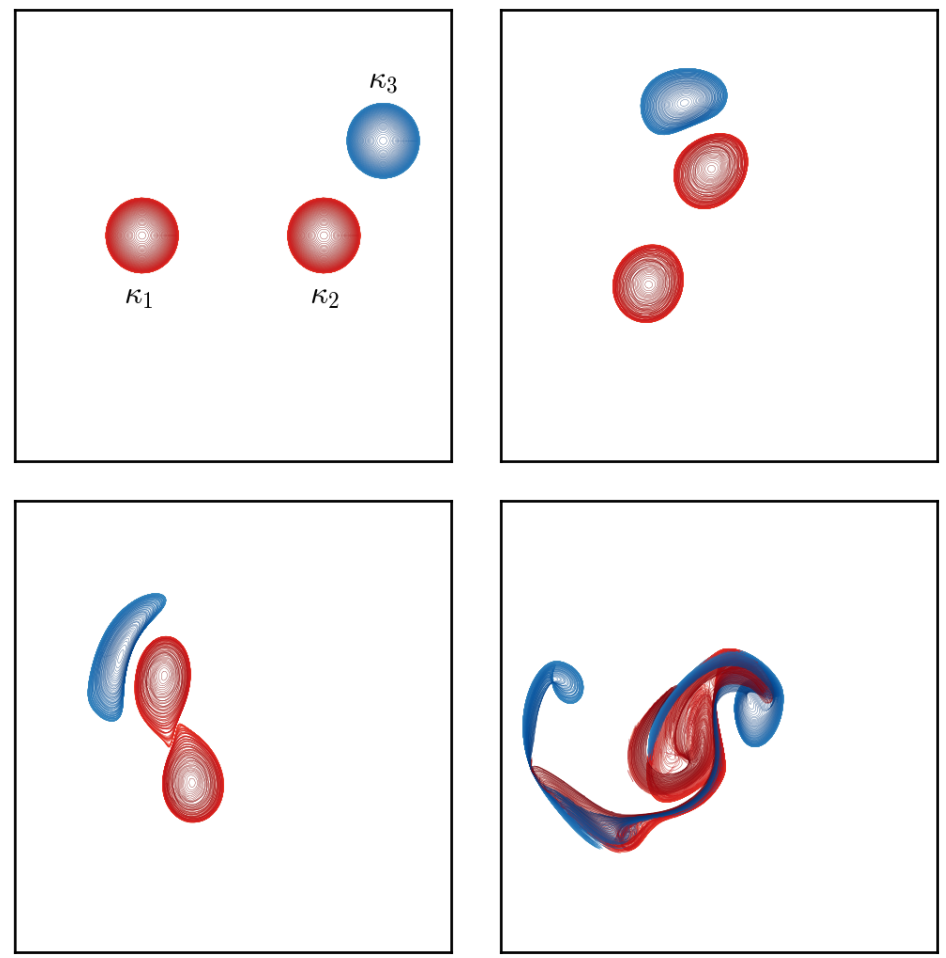

Figure 8. Top view on vortex bounding contours for three-dimensional QG vortices under the initial conditions corresponding to the self-similar collapse of equivalent point vortices for $\kappa_{1} / \kappa_{2}=1, s_{1}(0) / s_{3}(0)=0.6, r_{0} / s_{3}(0)=0.2$ at $t=0,15.5,20$ and 28 . The three vortices are labelled in the first panel. The region displayed horizontally is $-3 \leq x, y \leq 3$ (colour online).
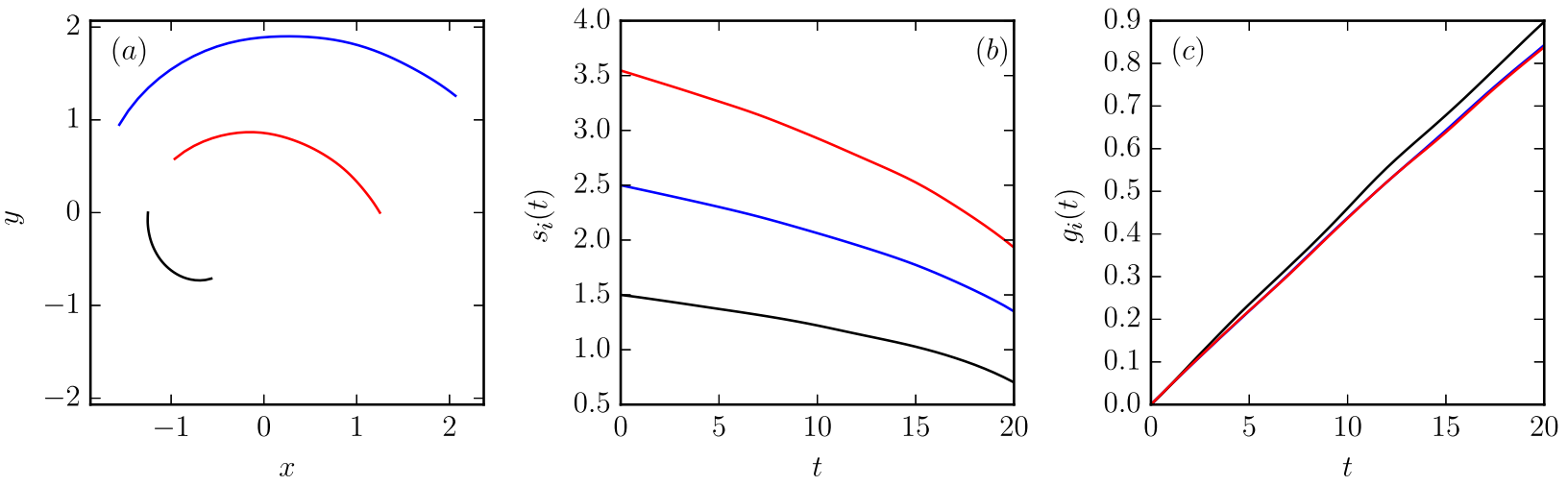

Figure 9. (a) Trajectories of the finite volume QG vortex centres under the initial conditions corresponding to the self-similar collapse of equivalent point vortices for $\kappa_{1} / \kappa_{2}=1, s_{1}(0) / s_{3}(0)=0.6, r_{0} / s_{3}(0)=0.2$, and for $0 \leq t \leq 20$, and $(b)$ the distances $s_{i}(t), i=1,2,3$ separating the centres, $(c)$ self-similarity function $g_{i}(t)$ (colour online).

the one predicted by the point vortex calculation, as one expects.

Figure 12 gives the relative volume $V_{i}(t) / V_{i}(0), i=1,2,3$ of the three largest vortices identified in the flow at any time $t$ for both $r_{0} / s_{3}(0)=0.2$, and $r_{0} / s_{3}(0)=0.1$ as a function of time. At $t=0$, all three vortices have the same volume. In both cases, the largest vortex first nearly doubles in size, indicating the merger of the two like-signed vortices. Indeed, in the figure, the black curve corresponding to the largest vortex, reaches $V_{i}(t) / V_{i}(0) \simeq 2$, and the blue curve corresponding to the smallest of the three largest vortices collapses to 0 . By the time of the merger stage, the opposite-signed vortex has retained almost all its volume. 

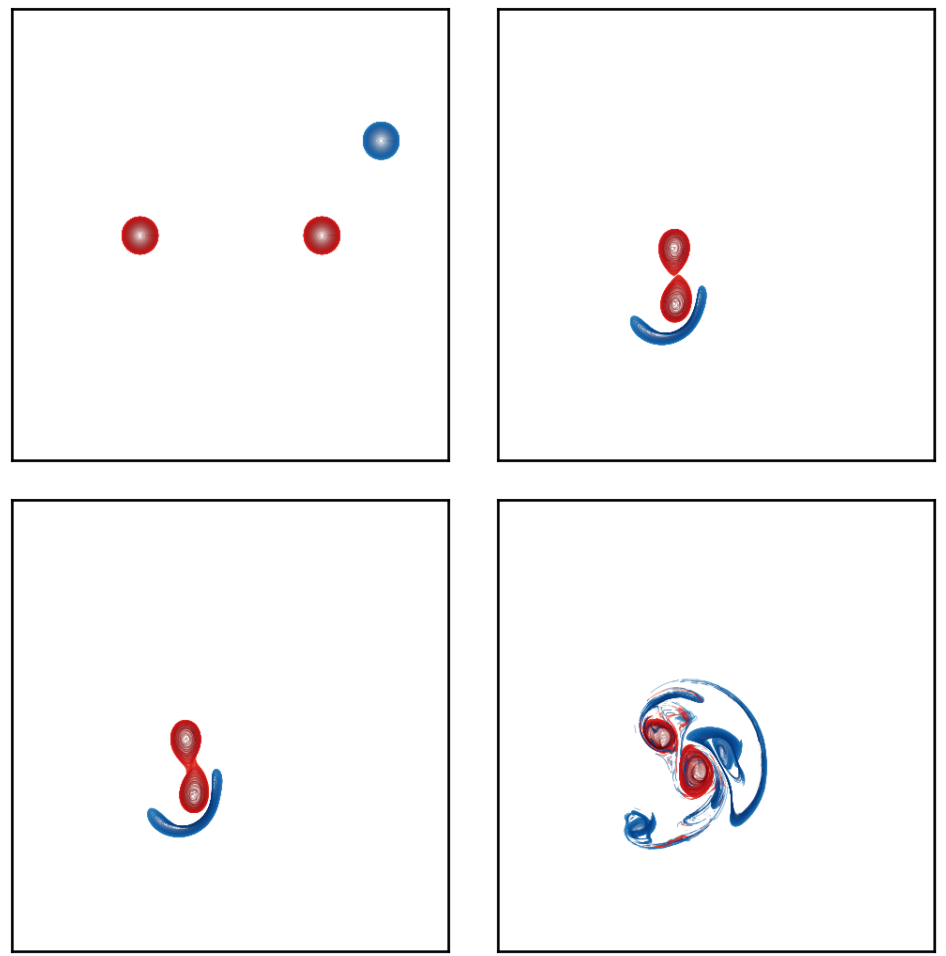

Figure 10. Top view on the vortex bounding contours for three-dimensional QG vortices under the initial conditions corresponding to the self-similar collapse of equivalent point vortices for $\kappa_{1} / \kappa_{2}, s / s_{3}(0)=0.6$ and $r_{0} / s_{3}(0)=0.1$ at $t=0,183,183.5$ and 206. The region displayed horizontally is $-6 \leq x, y \leq 6$ (colour online).
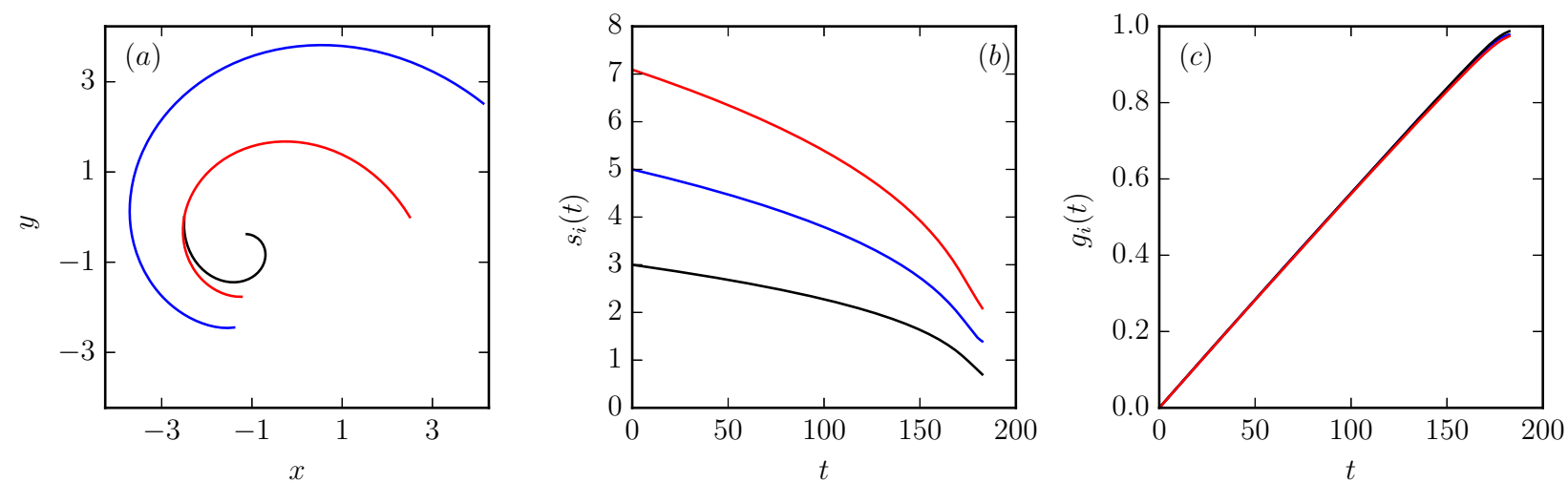

Figure 11. (a) Trajectories of the finite volume QG vortex centres under the initial conditions corresponding to the self-similar collapse of equivalent point vortices for $\kappa_{1} / \kappa_{2}, s_{1}(0) / s_{3}(0)=0.6$, and for $r_{0} / s_{3}(0)=0.1$ for $0 \leq t \leq 206,(b)$ the distances $s_{i}(t), i=1,2,3$ separating the vortex centres, and $(c)$ self-similarity functions $g_{i}(t)$ (colour online).

It initially corresponds to the red curves in figure 12 . This vortex is however highly deformed and later shed a fraction of its volume. This vortex is thus partially destroyed and then breaks into the second and third largest vortices present in the flow (see the red and blue curves). The later evolution is more complex. The evolution of the volume of the largest vortex (black curve in figure 12) shows that the merged vortex eventually loses material. The outcome of the interaction is therefore a partial merger as observed from the flow snapshots.

Finally, we evaluate the impact of the interaction on the amount of enstrophy

$$
E_{v}=\frac{1}{2} \iiint q^{2} \mathrm{~d}^{3} \boldsymbol{x},
$$



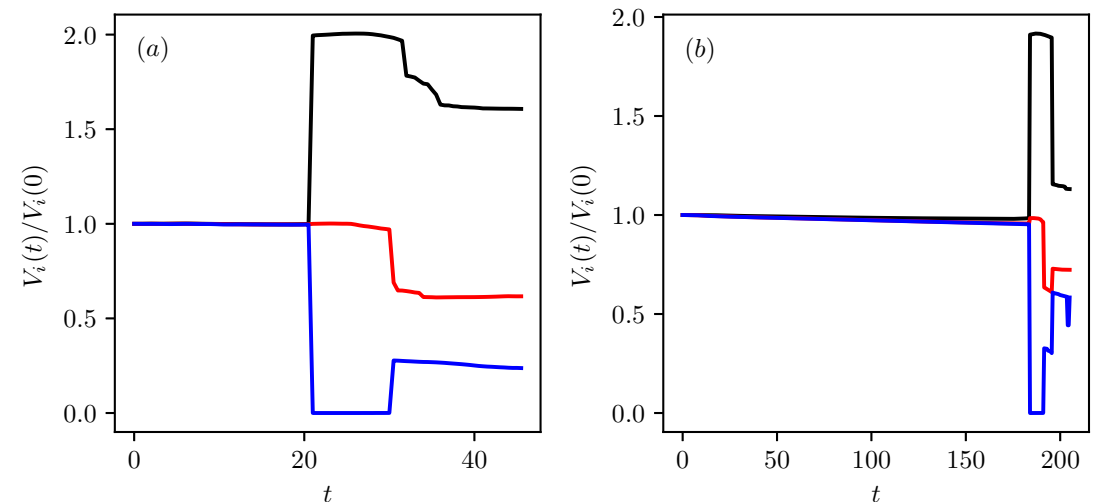

Figure 12. Evolution of the volume $V_{i}(t), i=12,3$ of the three largest vortices in the flow for finite volume QG vortices under the initial conditions corresponding to the self-similar collapse of equivalent point vortices for $\kappa_{1} / \kappa_{2}=1$, $s / s_{3}(0)=0.6$ and $(a): r_{0} / s_{3}(0)=0.2,(b): r_{0} / s_{3}(0)=0.1$ (colour online).
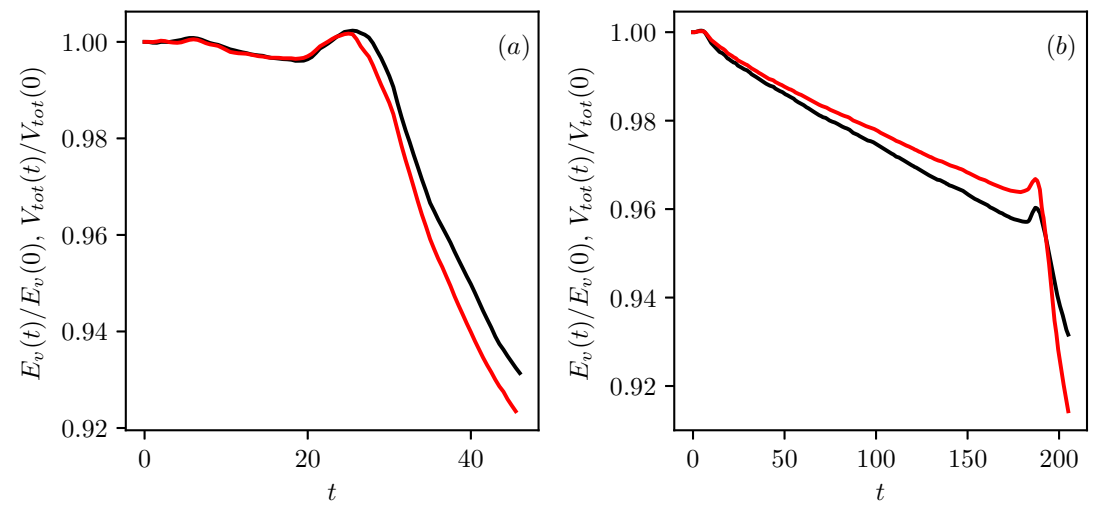

Figure 13. Evolution of the dimensionless enstrophy $E(t) / E(0)$ (black) and dimensionless total PV volume $V_{\text {tot }}(t) / V_{\text {tot }}(0)$ (red) for finite volume QG vortices under the initial conditions corresponding to the self-similar collapse of equivalent point vortices for $\kappa_{1} / \kappa_{2}=1, s / s_{3}(0)=0.6$ and $(a): r_{0} / s_{3}(0)=0.2,(b): r_{0} / s_{3}(0)=0.1(\operatorname{colour}$ online).

in the flow. Since the vortices consist of materially-conserved uniform PV, $E_{v}$ is closely linked to the volume of PV present in the flow. This volume is conserved but for the loss of material associated with the removal, by contour surgery, of the smallest (dynamically unimportant) debris and filaments. Contour surgery indeed mimics the dissipation of the finest scales in the flow. The loss of material is therefore directly linked to the production of small scales and the associated direct cascade of enstrophy. Gotoda and Sakayo (2018) indicates that vortex collapse provides a mechanism to dissipate enstrophy in two-dimensional turbulence. The time evolution of the enstrophy $E_{v}$ and the total volume $V_{t o t}$ of $\mathrm{PV}$ present in the flow are given in figure 13. The merger of the like-signed vortices generates a large amount of small scale debris and filaments. These debris and filaments are then strained, stretched and broken into smaller pieces. Their size shrinks and cascades downward, until they are removed by Contour Surgery. The interaction has therefore precipitated the 'dissipation' of enstrophy.

\section{Example of the collapse for finite area SQG vortices}

We finally turn our attention to the interaction of three SQG vortices. In the previous calculations, the vortices at $t=0$ were spheres of uniform PV. Such vortices have a linear internal 


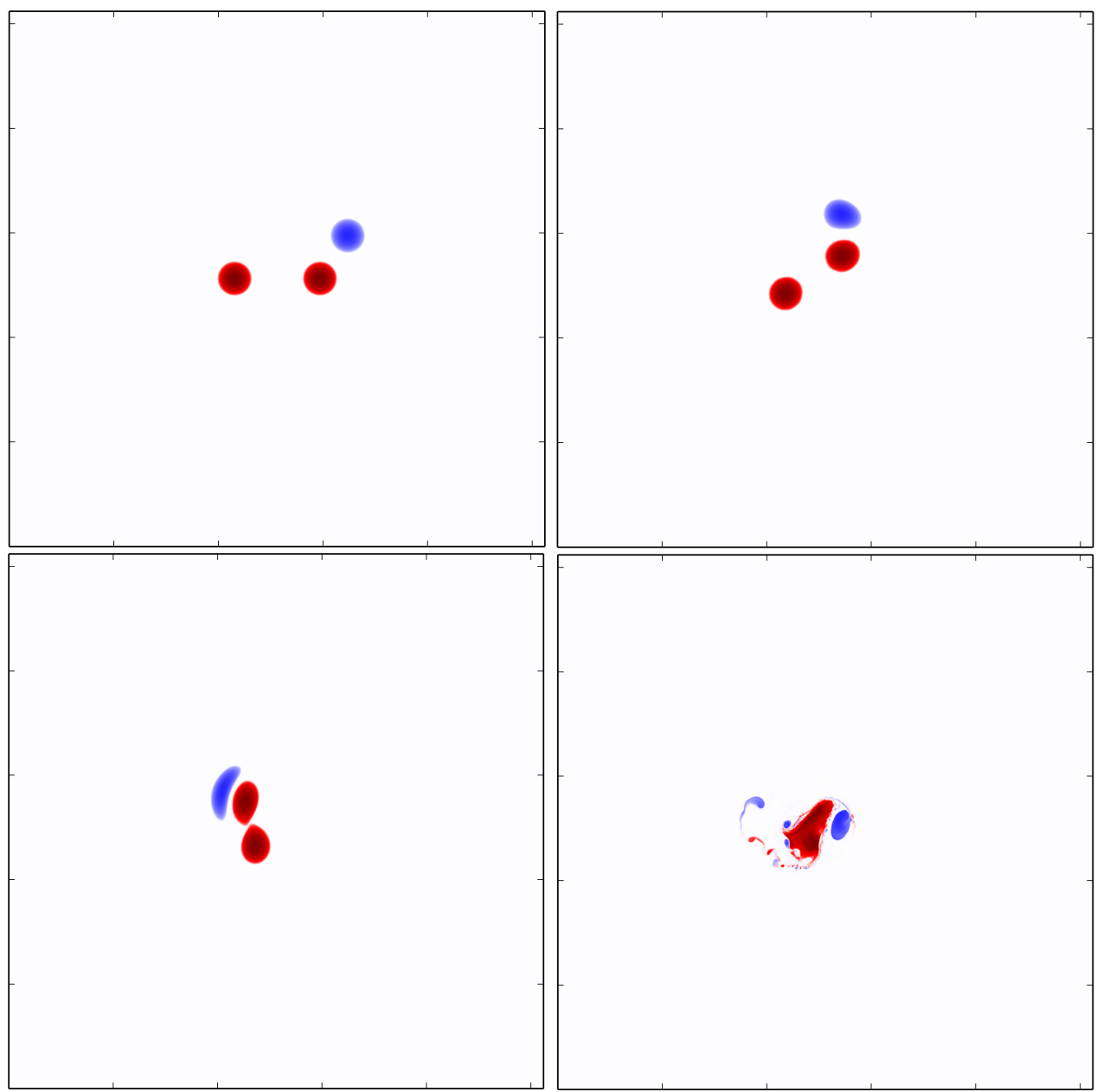

Figure 14. Snapshot of the buoyancy $\tilde{b}$ for parabolic SQG vortices in the condition of self-similar collapse of equivalent point vortices for $\kappa_{1} / \kappa_{2}=1, s_{1}(0) / s_{3}(0)=0.6$, for $r_{0} / s_{3}(0)=0.2$ and at $t=0,12.5,22.25$ and 28 in the full computational domain $[-\pi, \pi]^{2}$ (colour online).

velocity field. Vortices with a linear internal velocity field in SQG have a 'parabolic' buoyancy distribution as discussed by Dritschel (2011).

The buoyancy associated with vortex $i$ of radius $r_{i}$ and centred at $\left(x_{i}, y_{i}\right)$ is defined by

$$
\tilde{b}_{i}(\boldsymbol{x})= \begin{cases}\tilde{b}_{i}^{m} \sqrt{1-\left(\frac{r^{\prime}}{r_{i}}\right)^{2},}, & r^{\prime} \leq r_{i}, \\ 0, & r^{\prime}>r_{i} .\end{cases}
$$

where $r^{\prime}=\left(x-x_{i}\right)^{2}+\left(y-y_{i}\right)^{2}$ is the local radius. Again, we set $\kappa_{1}=\kappa_{2}$ and $s_{1}(0) / s_{3}(0)=$ 0.6. The simulations are performed using Combined-Lagrangian-Advection-Model (CLAM) developed by Dritschel and Fontane (2010). The domain is doubly-periodic, and of dimension $[-\pi, \pi]^{2}$. The resolution of the inversion grid on which the velocity field is determined is $1024^{2}$. The buoyancy field is represented by a combination of grid points or spectral coefficients (large scales), material contours (intermediate scales), and particles (small scales), down to scales as small as a $1 / 16$ of the inversion grid spacing. The effective resolution for the buoyancy field is $16,384^{2}$. We set $\tilde{b}_{1}^{m}=\tilde{b}_{2}^{m}=1, s_{3}(0)=1$. In the first experiment we $r_{1}=r_{2}=r_{3}=r_{0}=0.2$. The vortices only occupy a limited area near the centre of the full computational domain, keeping the influence of period images of the vortices low.

Snapshots of the flow are presented in figures 14 and 15 . The early evolution is similar 


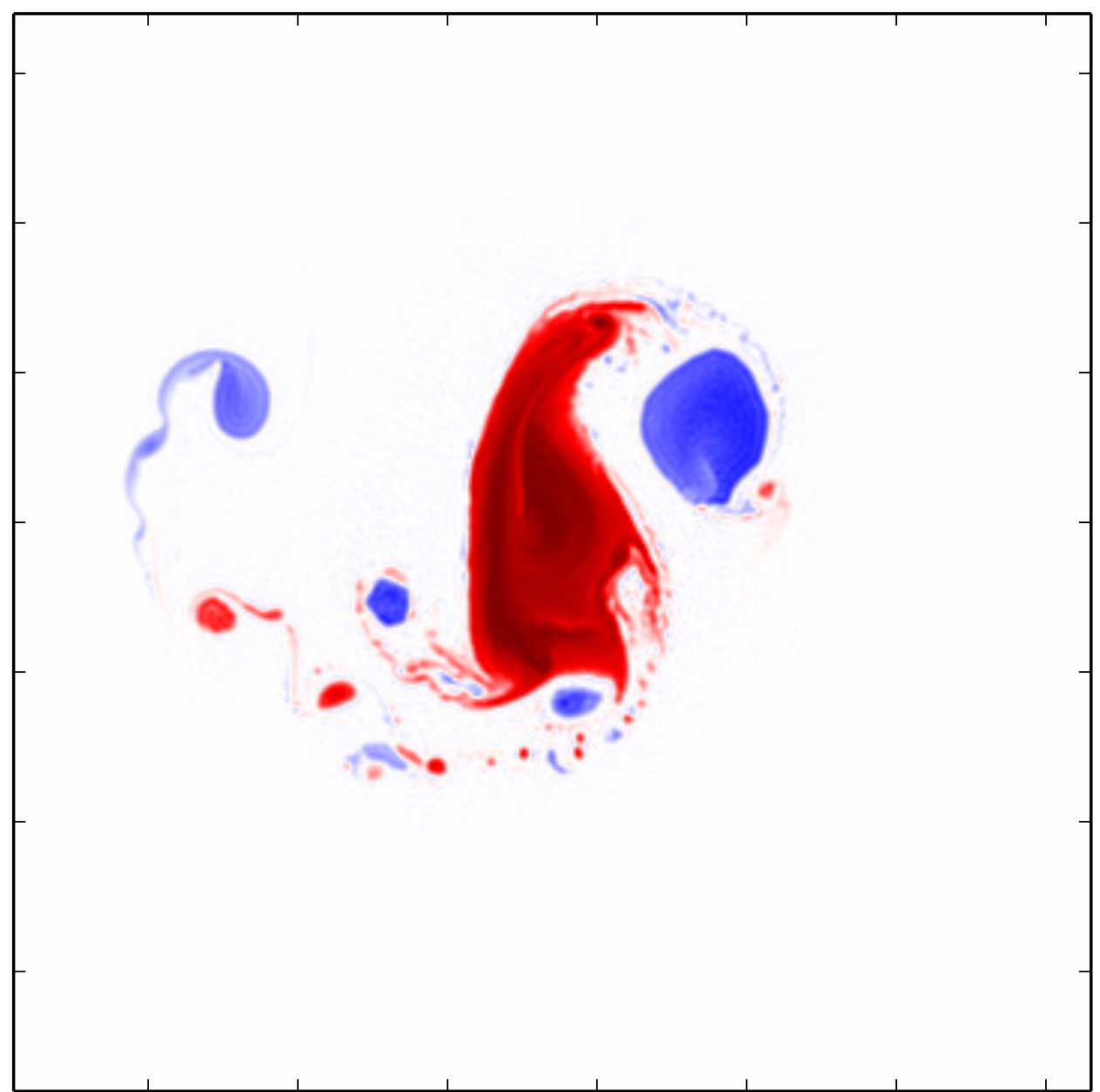

Figure 15. Close-up on the buoyancy $\tilde{b}$ for parabolic SQG vortices in the condition of self-similar collapse of equivalent point vortices for $\kappa_{1} / \kappa_{2}=1, s_{1} / s_{3}=0.6$ at $t=29$ in the domain $[-1.276,0.924] \times[-1.246,0.963]$ (colour online).

to the one of the three-dimensional QG vortices. The vortices spiral inward and get closer together. Then the two like-signed vortices merge at $t=22.5$. The later evolution differs qualitatively from the evolution of three-dimensional QG vortices and is specific to the SQG dynamics. The negative buoyancy vortex is sheared out by the merged, positive buoyancy vortex. It is stretched into a filament which rapidly breaks into a series of small scale eddies. The destabilisation of stretched filaments of buoyancy has been first analysed by Harvey and Ambaum (2010), and observed in many occasions, see for example Scott and Dritschel (2014), Reinaud et al. (2016). It differs from the evolution of a filament of PV. Indeed, the intensity of the shear in a filament of PV, as observed in the interaction of the three-dimensional QG vortices, is set by the PV $q$ which is materially conserved. Such a filament may remain stable when subjected to a large enough (stabilising) external strain, see Dritschel et al. (1991) for the two-dimensional case. On the other hand, the intensity of the shear in a filament of buoyancy depends on the ratio of the filament buoyancy $\tilde{b}$, which is materially conserved, to its thickness $a$. As the filament is stretched, its thickness $a$ decreases and the intensity of the shear in the filament increases. The shear always eventually overcomes the otherwise stabilising external strain, and the filament breaks down into small scale eddies through a shear instability similar to Kelvin-Helmholtz instability. This provides a rapid route towards a direct energy and enstrophy cascade.

Figure 16 shows the trajectories of the vortex centres, the distances $s_{i}(t)$ separating the vortices, and the self-similarity functions $g_{i}(t), i=1,2,3$ before the two like-signed vortices 

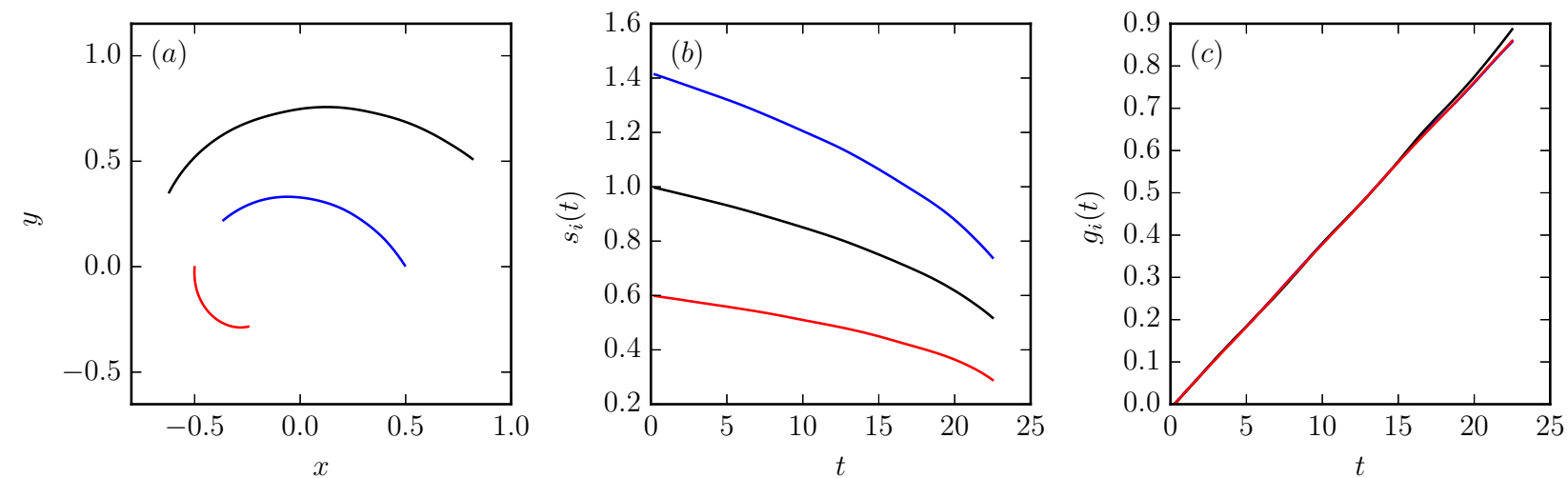

Figure 16. (a) Trajectories of the parabolic SQG vortex centres under the initial conditions corresponding to the selfsimilar collapse of equivalent point vortices for $\kappa_{1} / \kappa_{2}, s_{1}(0) / s_{3}(0)=0.6$, and for $r_{0} / s_{3}(0)=0.2$ for $0 \leq t \leq 22.5,(b)$ the distances $s_{i}(t), i=1,2,3$ separating the vortex centres, and $(c)$ self-similarity functions $g_{i}(t)$ (colour online).

merge. The vortices are identified from the full gridded combined buoyancy field as contiguous regions of buoyancy $\tilde{b}$ in excess (in absolute value) of twice the root mean squared buoyancy over the full periodic domain. The quasi linearity of the functions $g_{i}(t)$ confirms the early self-similar motion of vortex centres, with a cubic root time power law.

In the second experiment we set $r_{1}=r_{2}=r_{3}=0.1$. Snapshots of the flow evolution are shown in figure 17. As in the previous case, the vortices spiral inwards and the two like-signed vortices merger at $t \simeq 105$. The early self-similar motion is again confirmed in figure 18 which shows the trajectories of the vortex centres, the distances $s_{i}(t)$ between them, and the self-similarity functions $g_{i}(t)$. The latter functions are almost exactly linear.

Figure 19 shows the evolution of the equivalent enstrophy

$$
E_{b}=\frac{1}{2} \iint \tilde{b}^{2} \mathrm{~d}^{2} \boldsymbol{x}
$$

contained in the flow. The equivalent enstrophy is calculated from the full gridded combined buoyancy field. Note that this quantity is equivalent to an energy in SQG. Results show that the merger of the two like-signed vortices is associated in both cases with a strong and rapid dissipation of the enstrophy. This is due to the rapid breaking down of the buoyancy filaments discussed above. The system rapidly settles down to meta-stable a collection of eddies of various size.

Figure 20 shows the evolution of the surface area $A_{\max }(t)$ of the largest vortex present in the flow. Results clearly show the initial merger of the two like-signed vortices in both cases. The merged vortex then loses material by partially breaking out and shedding filamentary material. It eventually settles as a vortex whose size is roughly $50 \%$ larger than the size of the initial vortices.

\section{Conclusion}

We have studied a class of interactions between two like-signed vortices and an opposite-signed vortex. Three-vortex interactions have been observed in the oceans, see for example Carton et al. (2002). Rodríguez-Marroyo et al. (2011) reported the merger of two anticyclonic eddies in the vicinity of a cyclonic eddy, south of the Canary Islands. In this case, the vortices were very close together when they were observed, and it is not possible to ascertain whether the three vortices were in a self-similar collapse configuration. Nonetheless, as stressed by the authors, the presence of the cyclonic eddy precipitates the merger of the anticyclonic eddies. 

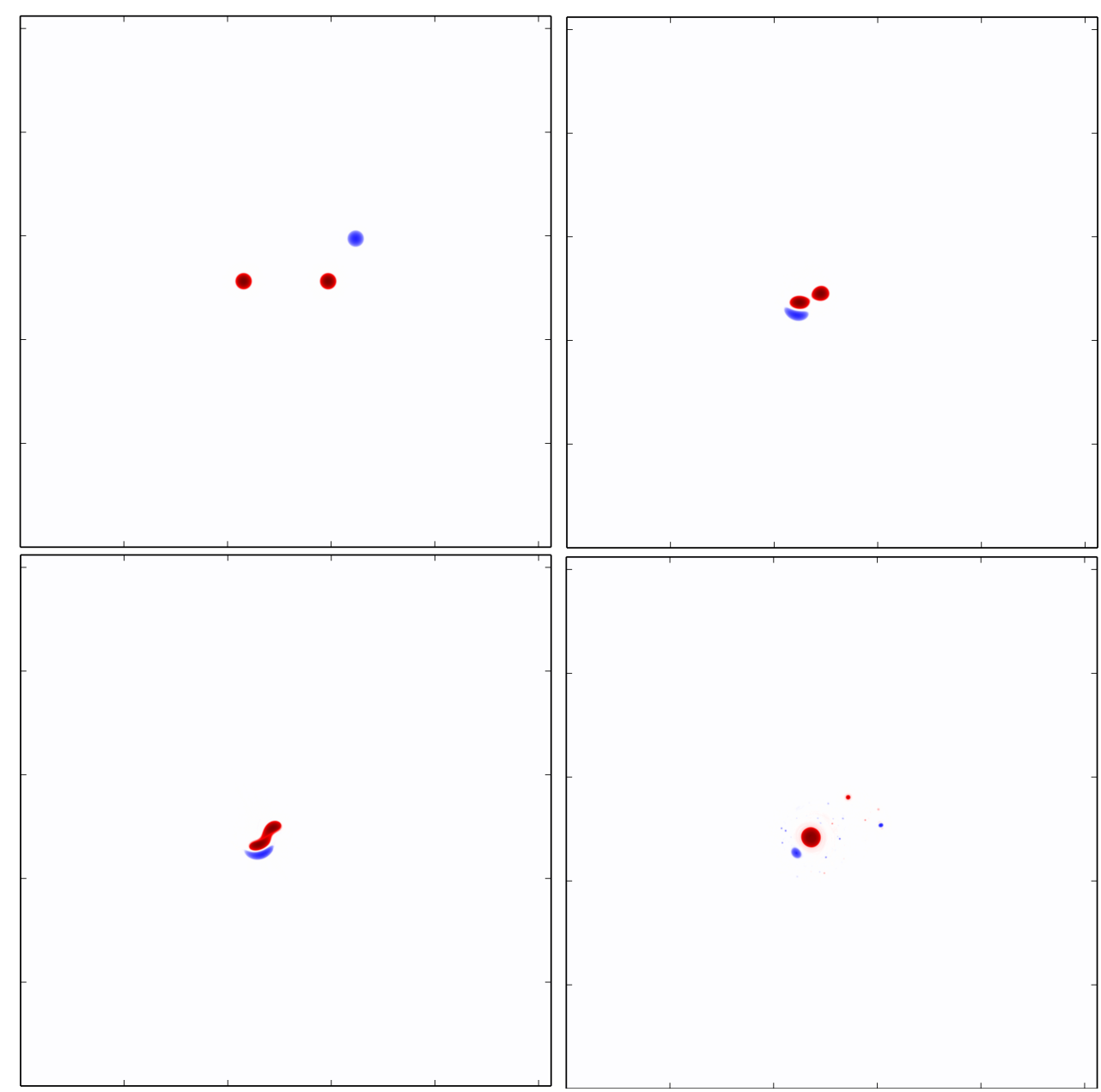

Figure 17. Snapshot of the buoyancy $\tilde{b}$ for parabolic SQG vortices in the condition of self-similar collapse of equivalent point vortices for $\kappa_{1} / \kappa_{2}=1, s_{1} / s_{3}=0.6$, and $r_{0} / s_{3}(0)=0.1$, and at $t=0,104.75,105.5$ and 200 in the full computational domain $[-\pi, \pi]^{2}$ (colour online).
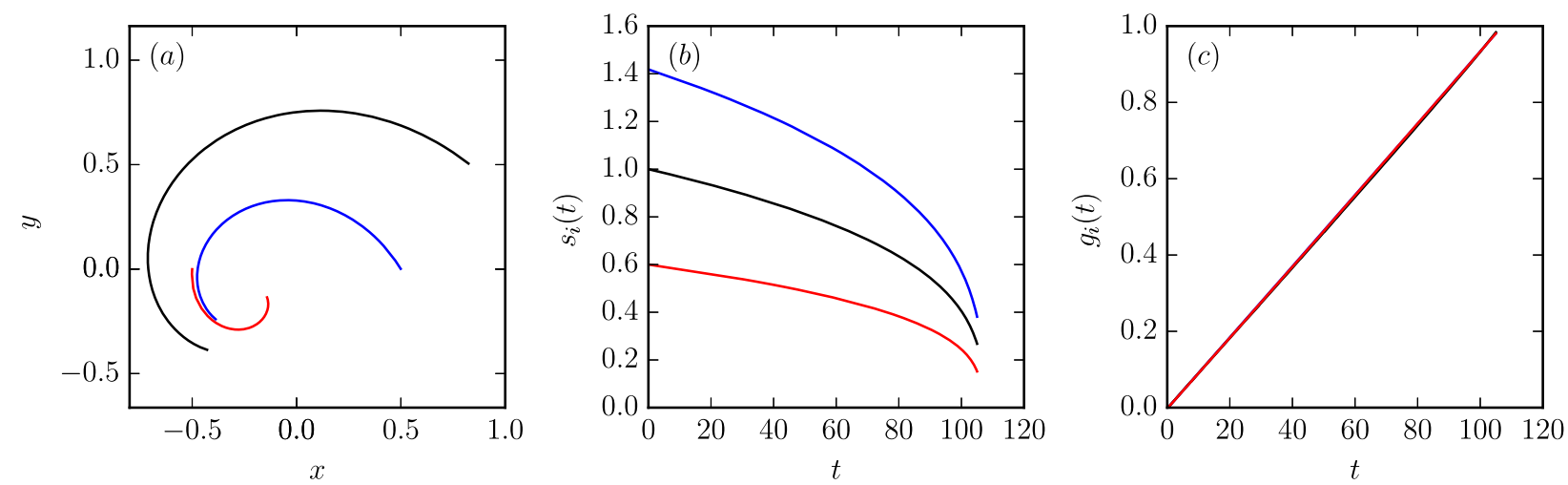

Figure 18. (a) Trajectories of the parabolic SQG vortex centres under the initial conditions corresponding to the selfsimilar collapse of equivalent point vortices for $\kappa_{1} / \kappa_{2}, s_{1}(0) / s_{3}(0)=0.6$, and for $r_{0} / s_{3}(0)=0.2$ for $0 \leq t \leq 104.75,(b)$ the distances $s_{i}(t), i=1,2,3$ separating the vortex centres, and $(c)$ self-similarity functions $g_{i}(t)$ (colour online). 

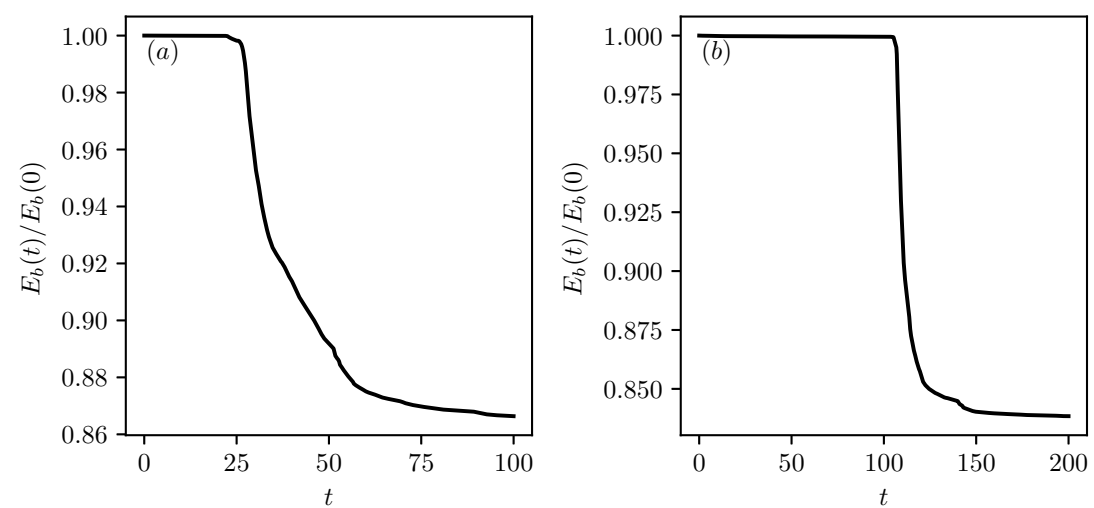

Figure 19. Evolution of the dimensionless entrosphy $E(t) / E(0)$ for parabolic SQG vortices under the initial conditions corresponding to the self-similar collapse of equivalent point vortices for $\kappa_{1} / \kappa_{2}=1, s / s_{3}(0)=0.6$ and $(a): r_{0} / s_{3}(0)=0.2$, $(b): r_{0} / s_{3}(0)=0.1$ (colour online).
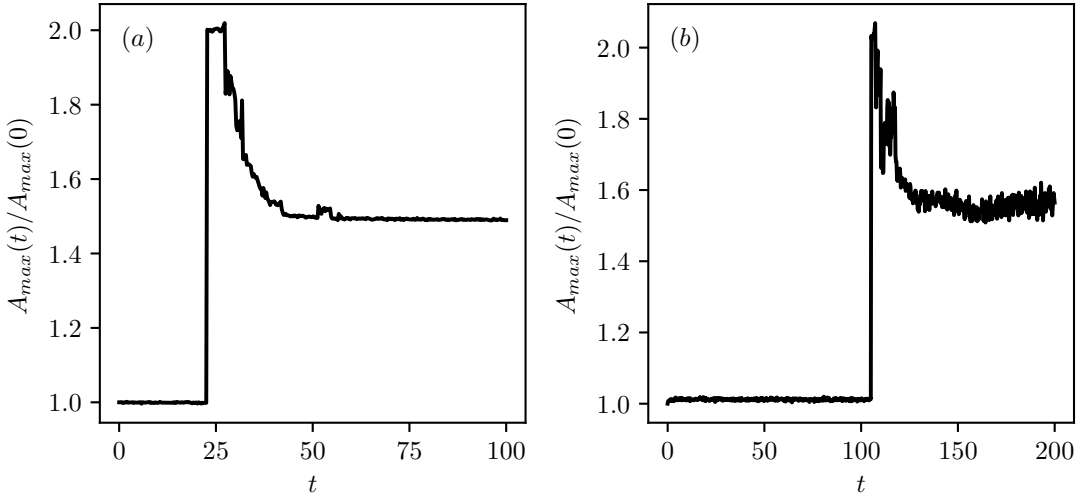

Figure 20. Evolution of the surface area $A_{\max }$ of the largest vortex for parabolic SQG vortices under the initial conditions corresponding to the self-similar collapse of equivalent point vortices for $\kappa_{1} / \kappa_{2}=1, s / s_{3}(0)=0.6$ and $(a)$ : $r_{0} / s_{3}(0)=0.2,(b): r_{0} / s_{3}(0)=0.1$.

Vortices only merge if they are close enough together. Recall that the distance between two interacting point vortices is invariant in the absence of external forcing. Hence two distant like-signed vortices do not, by themselves, move towards each other. The presence of a third interacting opposite-signed vortex allows the vortices to get closer together.

In this paper, we have shown that the concept of vortex collapse for a system of three two-dimensional point vortices generalises naturally to three-dimensional QG vortices and two-dimensional SQG vortices. The collapse is a consequence of a self-similar motion for the point vortices where the distances separating them collapse to zero, generating a singularity at finite time. This singularity, is is fact associated with the dynamics of point, i.e. singular vortices. Finite core vortices of same strength do not collapse onto a single point. Yet, they move towards each other along similar inward spiralling trajectories. This, in turn, favours the merger of the two like-signed vortices by reducing the distance separating the them. On the other hand, and at least when the two like-signed vortices have comparable strength, the weaker opposite-signed vortex is partially or totally destroyed. The interaction generates larger scales through the vortex merger as well as smaller scales through filamentation and straining out of the weaker vortex. We have also shown that these interactions may also be a source of strong enstrophy dissipation in geophysical flows.

We have considered the merger of two like-signed vortices, under the initial conditions 
determined by a point vortex calculation. This merger is possible if the finite core vortices follow closely the motion of the equivalent collapsing point vortices, at least until the two like-signed vortices are close enough to merge. It is however possible that the deformation of the vortices and the shedding of material due to their interaction, during the early stages of the evolution, make the vortices deviate strongly from the trajectory of the equivalent point vortices. A full investigation of the interaction between three finite core vortices should be considered in the future.

\section{References}

Aref, H., Motion of three vortices. Phys. Fluids, 1979, 22, 393-400.

Aref, H., Self-similar motion of three point vortices. Phys. Fluids, 2010, 22, 057104.

Carton, X., Chérubin, L., Paillet, J., Morel, Y., Serpette, A. and LeCann, B., Meddy coupling with a deep cyclone in the Gulf of Cadiz. J. Mar. Syst., 2002, 32, 13-42.

Charney, J.G., Geostrophic turbulence. J. Atmos. Sci., 1971, 28, 1087-1095.

Dritschel, D.G., Contour surgery: a topological reconnection scheme for extended integrations using contour dynamics. J. Comput. Phys., 1988, 77, 240-266.

Dritschel, D.G., An exact steadily rotating surface quasi-geostrophic elliptical vortex. Geophys. Astrophys. Fluid Dyn., 2011, 105, 368-376.

Dritschel, D.G. and Fontane, J., The combined Lagrangian advection method. J. Comput. Phys., 2010, 229, $5408-5417$.

Dritschel, D.G., Haynes, P.H., Juckes, M.N. and Shepherd, T.G., The stability of a two-dimensional vorticity filament under uniform strain. J. Fluid Mech., 1991, 230, 647-665.

Dritschel, D.G., Reinaud, J.N. and McKiver, W.J., The quasi-geostrophic ellipsoidal vortex model. J. Fluid Mech., 2004, 505, 201-223.

Dritschel, D.G. and Saravanan, R., Three-dimensional quasi-geostrophic contour dynamics, with an application to stratospheric vortex dynamics. Quart. J. Roy. Meteorol. Soc., 1994, 120, 1267-1297.

Gotoda, T. and Sakayo, T., Universality of the anomalous enstrophy dissipation at the collapse of three point vortices on Euler-Poincaré models. SIAM J. Appl. Math., 2018, 78, 2105-2128.

Gröbli, W., Spezielle Probleme über die Bewegung Geradliniger Paralleler Wirbelfäden, 1877 (Zürich: Druck von Zürcher und Furrer).

Harvey, B.J. and Ambaum, M.H.P., Instability of surface-temperature filaments in strain and shear. Q. J. Roy. Meteor. Soc. Part B, 2010, 136, 1506-1513.

Held, I.M., Pierrehumbert, R.T., Garner, S.T. and Swanson, K.L., Surface quasi-geostrophic dynamics. J. Fluid Mech., 1995, 282, 1-20.

Kimura, Y., Similarity solution of two-dimensional point vortices. J. Phys. Soc. Jpn., 1987, 56, 2024-2030.

Lapeyre, G., Surface Quasi-Geostrophy. Fluids, 2017, 2, 7.

Nastrom, G.D., Gage, K.S. and Jasperson, W.H., Kinetic energy spectrum of large-and mesoscale atmospheric processes. Nature, 1984, 310, 36-38.

Novikov, E.A., Dynamics and statistics of a system of vortices. Sov. Phys.-JETP, 1975, 41, 937-943.

Reinaud, J.N., Three-dimensional quasi-geostrophic vortex equilibria with $m$-fold symmetry. J. Fluid Mach. 2019, 863, 32-59.

Reinaud, J.N. and Dritschel, D.G., The merger of vertically offset quasi-geostrophic vortices. J. Fluid Mech. 2002, 469, 287-315.

Reinaud, J.N., Dritschel, D.G. and X., C., Interaction between a surface quasi-geostrophic buoyancy filament and an internal vortex. Geophys. Astrophys. Fluid Dyn., 2016, 110, 461-490.

Rodríguez-Marroyo, R., Viúdez, A. and Ruiz, S., Vortex merger in oceanic tripoles. J. Phys. Oceanogr., 2011, 41, 1239-1251.

Scott, R.K. and Dritschel, D.G., Numerical simulation of a self-similar cascade of filament instabilities in the surface quasigeostrophic system. Phys. Rev. Lett., 2014, 112, 144505.

Synge, J.L., On the motion of three vortices. Can. J. Math., 1949, 1, 257-270.

Tavantzis, J. and Ting, L., The dynamics of three vortices revisited. Phys. Fluids, 1988, 31, 1392-1409.

Vallis, G.K., Atmospheric and oceanic fluid dynamics: fundamentals and large-scale circulation, 2006 (Cambridge: Cambridge University Press).

Zhang, Z., Wang, W. and Qiu, B., Oceanic mass transport by mesoscale eddies. Science, 2014, 345, 322-324. 